\title{
Assessing the Strength of Directed Influences Among Neural Signals: An Approach to Noisy Data
}

\author{
Linda Sommerlade ${ }^{\mathrm{a}, \mathrm{b}, \mathrm{c}, \mathrm{d}, \mathrm{e}, *}$, Marco Thiel ${ }^{\mathrm{a}, \mathrm{b}}$, Malenka Mader ${ }^{\mathrm{c}, \mathrm{d}, \mathrm{f}}$, Wolfgang \\ Mader $^{\mathrm{c}, \mathrm{d}}$, Jens Timmer ${ }^{\mathrm{c}, \mathrm{d}, \mathrm{g}}$, Bettina Platt ${ }^{\mathrm{h}}$, Björn Schelter ${ }^{\mathrm{a}, \mathrm{b}, \mathrm{c}, * *}$ \\ ${ }^{a}$ Institute for Complex Systems and Mathematical Biology, University of Aberdeen, King's \\ College, Old Aberdeen AB24 3UE, United Kingdom \\ ${ }^{b}$ Institute for Pure and Applied Mathematics, University of Aberdeen, King's College, Old \\ Aberdeen AB24 3UE, United Kingdom \\ ${ }^{c}$ Freiburg Center for Data Analysis and Modeling (FDM), University of Freiburg, \\ Eckerstrasse 1, 79104 Freiburg, Germany \\ ${ }^{d}$ Institute for Physics, University of Freiburg, Hermann-Herder-Strasse 3a, 79104 Freiburg, \\ Germany \\ ${ }^{e}$ Freiburg Institute for Advanced Studies (FRIAS), University of Freiburg, Albertstrasse 19, \\ 79104 Freiburg, Germany \\ ${ }^{f}$ Department of Neuropediatrics and Muscular Disease, University Medical Center of \\ Freiburg, Mathildenstrasse 1, 79106 Freiburg, Germany \\ ${ }^{g}$ BIOSS Centre for Biological Signalling Studies, University of Freiburg, Schänzlestr. 18, \\ 79104 Freiburg, Germany \\ ${ }^{h}$ Institute of Medical Sciences, University of Aberdeen, Foresterhill, Aberdeen AB25 2ZD, \\ United Kingdom
}

\begin{abstract}
Background

Measurements in the neurosciences are afflicted with observational noise. Grangercausality inference typically does not take this effect into account. We demonstrate that this leads to false positives conclusions and spurious causalities.

New Method

State space modelling provides a convenient framework to obtain reliable estimates for Granger-causality. Despite its previous application in several studies, the analytical derivation of the statistics for parameter estimation in the state space model was missing. This prevented a rigorous evaluation of the results.
\end{abstract}

\footnotetext{
${ }^{*}$ Corresponding author

**Principal corresponding author

Email addresses: 1.sommerlade@abdn.ac.uk (Linda Sommerlade), m.thiel@abdn.ac.uk (Marco Thiel), Malenka.Mader@fdm.uni-freiburg.de (Malenka Mader),

Wolfgang.Mader@fdm.uni-freiburg.de (Wolfgang Mader), JeTi@fdm.uni-freiburg.de (Jens Timmer), b.platt@abdn.ac.uk (Bettina Platt), b.schelter@abdn.ac.uk (Björn Schelter)
} 
Results

In this manuscript we derive the statistics for parameter estimation in the state space model. We demonstrate in an extensive simulation study that our novel approach outperforms standard approaches and avoids false positive conclusions about Granger-causality.

Comparison with Existing Methods

In comparison with the naive application of Granger-causality inference, we demonstrate the superiority of our novel approach. The wide-spread applicability of our procedure provides a statistical framework for future studies. The application to mice electroencephalogram data demonstrates the immediate applicability of our approach.

\section{Conclusions}

The analytical derivation of the statistics presented in this manuscript enables a rigorous evaluation of the results of Granger causal network inference. It is noteworthy that the statistics can be readily applied to various measures for Granger causality and other approaches that are based on vector autoregressive models.

Granger-causality, Observational Noise, Statistics, Expectation-Maximisation Algorithm, Kalman Filter, Incomplete Data Likelihood, Analytical Covariance Matrix

\section{Introduction}

Complex systems are relevant in different branches of physics, economics, sociology, biology or the neurosciences. They can be investigated either by explicit first principle modelling of the dynamics, the so-called direct approach, or by inferring the system based on observed data, i.e. the inverse approach. The first requires profound a priori knowledge about the investigated system while the second relies on measurements of the system.

Within the framework of data-based modelling, various techniques have been developed. For many years, the methodological developments and concepts 
evolved independently in the different disciplines. In the past decade however, the transfer of knowledge across the borders of the different research fields has increased substantially, for example when methods dealing with non-linear stochastic systems have been introduced (Schelter et al., 2006).

Networks of interacting nodes, each with its own dynamics, are a key mathematical tool for the description of complex systems (Strogatz, 2001). Depending on the particular application, the dynamics of the individual nodes, their coupling structure or their collective behaviour all determine the dynamics of the system. In the neurosciences, for instance, detecting interactions between signals, i.e. the coupling structure among nodes, is of particular interest. Understanding brain networks promises to disclose the biological basis underlying natural behaviour or certain diseases (e.g. Hesse et al., 2003; Tass et al., 1998; Pitzalis et al., 1998; Keyl et al., 2000; Nollo et al., 2005; Bowers and Murray, 2004). Several techniques have been proposed so far to infer the network structure of complex systems from observed signals. These include but are not limited to transfer entropy (Schreiber, 2000; Staniek and Lehnertz, 2008), recurrences in state space (Arnhold et al., 1999; Chicharro and Andrzejak, 2009; Romano et al., 2007), mutual information (Pompe et al., 1998; Paluš and Stefanovska, 2003; Paluš and Vejmelka, 2007; Vejmelka and Paluš, 2008; Frenzel and Pompe, 2007), phase dynamics (Rosenblum and Pikovsky, 2001; Rosenblum et al., 2002), coherence (Halliday and Rosenberg, 2000; Dahlhaus, 2000; Nolte et al., 2008), the Fokker Planck formalism (Prusseit and Lehnertz, 2008; Bahraminasab et al., 2009), or autoregressive modelling (Dahlhaus and Eichler, 2003; Schack et al., 1995; Eichler, 2000; Korzeniewska et al., 1997; Kamiński et al., 1997; Kamiński and Blinowska, 1991; Arnold et al., 1998).

When investigating interactions among processes, cross-spectral analysis is often chosen (Brockwell and Davis, 1998). If the number of processes exceeds two, the question arises, whether interactions are direct or indirect. To address this challenge, the concept of partialisation is used (Dahlhaus, 2000; Brillinger, 1981). Partialisation aims at revealing direct connections by subtracting influences of third processes (Schad et al., 2009). 
Since (partial) cross-spectra are Hermitian, it is not possible to infer the direction of an influence from coherences. Additional information of the complex valued cross-spectra can be reveal by analysing their phases. The phase spectra can be used to draw conclusions about the direction of an influence. However, these conclusions are hampered if, for instance, filters are present. Since filters are present in many applications, a straight forward interpretation of the phase spectra is usually not possible. Other approaches to investigate the direction of an influence use the concept of causality. Many methods (e.g. Hesse et al., 2003; Geweke, 1982, 1984; Chen and Wasterlain, 2006; Dhamala et al., 2008; Baccalá and Sameshima, 2001; Sameshima and Baccalá, 1999; Eichler, 2006; Kamiński and Blinowska, 1991) are based on Granger's definition of causality (Granger, 1969). Briefly, this definition states that a process $x_{1}$ is causal for another process $x_{2}$, if $x_{1}$ is useful for the prediction of the future of $x_{2}$. Linear Granger-causality is typically modelled by means of vector autoregressive processes, which are estimated via multivariate Yule-Walker equations or similar approaches (Lütkepohl, 1993).

The estimators for the vector autoregressive models do not account for observational noise, which afflicts almost any observed signal. There are different reasons for an inaccurate measurement, such as the precision of the measurement device or influences from the environment. This inevitably leads to a misestimation of the parameters; typically parameters are underestimated (Brockwell and Davis, 1998). We emphasize that in particular an over-estimation of certain parameters has a severe impact on Granger-causality analysis. As also discussed in (Newbold , 1978; Nalatore et al., 2007; Nolte et al., 2008), we argue that this leads to spurious causalities independently of the specific measure for Granger-causality used, providing a more detailed view on the relation between parameter values and the severity of the misestimation for various signal to noise ratios. This explains in particular how under- as well as over-estimation of parameters leads to spurious interactions. Based on analytical calculations we reveal the cause of these spurious causalities. To this aim, we discuss a vector autoregressive process of order 2 , to simplify the notation. We then investigate 
in details the consequences of the misestimation. We will also show that increasing the order of the fitted process yields an improved estimator. However, this is not sufficient to avoid false positive conclusions in the presence of observational noise (Timmer, 1998). Therefore, in this manuscript, state space modelling is used to explicitly include observational noise in the model. The idea of state space modelling for improving measures quantifying Granger-causality has been introduced in (Winterhalder et al., 2005). This triggered further research that discussed the matter in more detail (Nalatore et al., 2007) and also an application to neurophysiological data (Nalatore et al., 2009). The corresponding statistics to assess the statistical significance of these Granger-causality measures, however, has not been thoroughly investigated. Typically Monte-Carlo based procedures, numerical approximations or bootstrap based approaches are employed to approximate the statistics for these measures. In this manuscript we assume that the system under investigation can be approximated by a linear system. Based on this assumption, we derive an analytical statistics for Granger-causality based measures.

Throughout, we demonstrate our results for a specific measure for Grangercausality, the so-called renormalised partial directed coherence (rPDC) (Schelter et al., 2009). It is a generalisation of partial directed coherence, which was introduced to quantify Granger causal influences (Baccalá and Sameshima, 2001) in the neurosciences (Sameshima and Baccalá, 1999; Nicolelis and Fanselow, 2002). The advantage of renormalised partial directed coherence is that it allows us to interpret the results also in terms of the strengths of interactions and it enables a rigorous comparison of the results. We emphasise though that the results apply to any Granger-causality measure that is based on vector autoregressive processes. The results are presented in a way that they can readily be generalised.

\section{Observational Noise and rPDC}

To demonstrate the effects of observational noise on the reconstructed interaction structure, the two dimensional vector autoregressive process of order 2 
(VAR [2] process)

$$
\begin{gathered}
\vec{x}(t)=\sum_{r=1}^{2} \mathbf{a}_{r} \vec{x}(t-r)+\varepsilon_{x}(t) \\
y_{i}(t)=x_{i}(t)+\sigma_{i} \eta_{i}(t) \quad i=1,2
\end{gathered}
$$

with $\varepsilon_{x}$ and $\eta_{i}$ standard Gaussian white noise and

$$
\mathbf{a}_{1}=\left(\begin{array}{cc}
1.3 & c \\
0 & 1.7
\end{array}\right), \mathbf{a}_{2}=\left(\begin{array}{cc}
-0.8 & 0 \\
0 & -0.8
\end{array}\right)
$$

is investigated under the assumption that only $\vec{y}$ can be measured. Thus, only a noisy version of the true process $\vec{x}$ is available to examine the interaction structure. The causal influence from process $x_{2}$ onto $x_{1}$ is represented by the parameter $c$ in this model and set to $c=0.3$. The $\sigma_{i}$ are chosen such that different noise-to-signal ratios are achieved. This ratio between the noise variance and the process variance is varied between 0 and 1 in steps of 0.05 for each component. For each combination of noise-to-signal ratios 100 realisations are simulated with $N=5,000$ data points each. Renormalised partial directed coherence (see Appendix A) is estimated for every realisation. Results for $p=2$ are shown in Fig. 1. For each combination of noise-to-signal ratios the percentage of realisations with significant renormalised partial directed coherence at the oscillation frequencies of 0.12 and $0.05 \mathrm{~Hz}$, respectively, is shown. The influence from process $x_{2}$ onto $x_{1}$ which is present in the simulation is always detected correctly (Fig. 1 right). Additionally the direction from process $x_{1}$ onto $x_{2}$ is also significantly different from zero for many realisations if process $x_{2}$ is afflicted with observational noise (Fig. 1 left).

In order to understand the results in more detail, the influence of observational noise on the parameter estimation is investigated. A frequently used parameter estimator for autoregressive processes is (Lütkepohl, 1993)

$$
\hat{\mathbf{a}}_{l}=\sum_{m=1}^{p}(\hat{\mathbf{R}})^{-1}(l, m) \hat{\mathbf{r}}(m)
$$



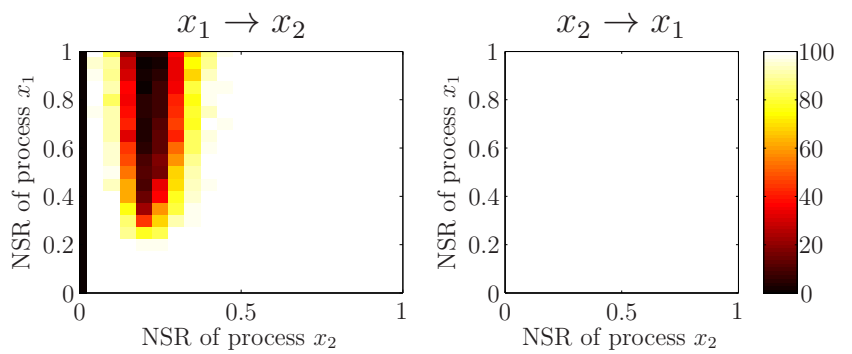

Figure 1: Percentage of realisations with significant renormalised partial directed coherence at the oscillation frequencies of the driving process, 0.12 and $0.05 \mathrm{~Hz}$, respectively. For the estimation, a model order of $p=2$ is used.

with

$$
\hat{\mathbf{R}}(l, m)=\frac{1}{N-p} \sum_{t=p+1}^{N} \mathbf{x}(t-l) \mathbf{x}^{\prime}(t-m)
$$

and $\hat{\mathbf{r}}(m)=\hat{\mathbf{R}}(0, m) ; \mathbf{x}^{\prime}$ denotes transposition of $\mathbf{x}$. For an autoregressive process of order 2 (AR $[2]$ process), this leads to

$$
\begin{aligned}
& \hat{\mathbf{a}}_{1}=\hat{\mathbf{R}}^{-1}(1,1) \hat{\mathbf{r}}(1)+\hat{\mathbf{R}}^{-1}(1,2) \hat{\mathbf{r}}(2) \\
& \hat{\mathbf{a}}_{2}=\hat{\mathbf{R}}^{-1}(2,1) \hat{\mathbf{r}}(1)+\hat{\mathbf{R}}^{-1}(2,2) \hat{\mathbf{r}}(2) .
\end{aligned}
$$

In order to avoid complicated notations the one dimensional case is considered in the following. This yields

$$
\hat{\mathbf{R}}^{-1}=\frac{1}{\langle x(t), x(t)\rangle^{2}-\langle x(t), x(t-1)\rangle^{2}}\left(\begin{array}{cc}
\langle x(t), x(t)\rangle & -\langle x(t), x(t-1)\rangle \\
-\langle x(t), x(t-1)\rangle & \langle x(t), x(t)\rangle
\end{array}\right)
$$

and thus

$$
\begin{aligned}
& \hat{\mathbf{a}}_{1}=\frac{\langle x(t), x(t)\rangle\langle x(t), x(t-1)\rangle-\langle x(t), x(t-1)\rangle\langle x(t), x(t-2)\rangle}{\langle x(t), x(t)\rangle^{2}-\langle x(t), x(t-1)\rangle^{2}} \\
& \hat{\mathbf{a}}_{2}=\frac{\langle x(t), x(t)\rangle\langle x(t), x(t-2)\rangle-\langle x(t), x(t-1)\rangle^{2}}{\langle x(t), x(t)\rangle^{2}-\langle x(t), x(t-1)\rangle^{2}} .
\end{aligned}
$$

In all calculations, we assume ergodicity, such that the expectation values can be calculated with respect to time. If instead of the original data $x(t)$ a noisy 
observation $x_{\mathrm{obs}}(t)=x(t)+\eta(t)$ is used for the estimation, only $\left\langle x_{\mathrm{obs}}(t), x_{\mathrm{obs}}(t)\right\rangle$ differs from its noise free counterpart as

$$
\begin{aligned}
\left\langle x_{\mathrm{obs}}(t), x_{\mathrm{obs}}(t)\right\rangle & =(1+\mathrm{NSR})\langle x(t), x(t)\rangle \\
\left\langle x_{\mathrm{obs}}(t), x_{\mathrm{obs}}(t-1)\right\rangle & =\langle x(t), x(t-1)\rangle \\
\left\langle x_{\mathrm{obs}}(t), x_{\mathrm{obs}}(t-2)\right\rangle & =\langle x(t), x(t-2)\rangle
\end{aligned}
$$

with NSR $=\frac{\langle\eta(t), \eta(t)\rangle}{\langle x(t), x(t)\rangle}$, the noise-to-signal ratio. Inserting this in Eqn. (9) and (10) leads to

$$
\begin{aligned}
& \hat{\mathbf{a}}_{1}=\frac{(1+\mathrm{NSR})\langle x(t), x(t)\rangle\langle x(t), x(t-1)\rangle-\langle x(t), x(t-1)\rangle\langle x(t), x(t-2)\rangle}{(1+\mathrm{NSR})^{2}\langle x(t), x(t)\rangle^{2}-\langle x(t), x(t-1)\rangle^{2}} \\
& \hat{\mathbf{a}}_{2}=\frac{(1+\mathrm{NSR})\langle x(t), x(t)\rangle\langle x(t), x(t-2)\rangle-\langle x(t), x(t-1)\rangle^{2}}{(1+\mathrm{NSR})^{2}\langle x(t), x(t)\rangle^{2}-\langle x(t), x(t-1)\rangle^{2}}
\end{aligned}
$$

In order to eliminate the auto-covariances in these equations, they can be related to one another using the true parameters

$$
\begin{aligned}
\langle x(t), x(t-1)\rangle & =\frac{\mathbf{a}_{1}}{1-\mathbf{a}_{2}}\langle x(t), x(t)\rangle \\
\langle x(t), x(t-2)\rangle & =\frac{\mathbf{a}_{1}^{2}+\mathbf{a}_{2}-\mathbf{a}_{2}^{2}}{1-\mathbf{a}_{2}}\langle x(t), x(t)\rangle,
\end{aligned}
$$

see Appendix B for details. Inserting this in Eqn. (14) and (15) leads to

$$
\begin{aligned}
& \hat{\mathbf{a}}_{1}=\mathbf{a}_{1} \frac{(1+\mathrm{NSR})\left(1-\mathbf{a}_{2}\right)-\left(\mathbf{a}_{1}^{2}+\mathbf{a}_{2}-\mathbf{a}_{2}^{2}\right)}{(1+\mathrm{NSR})^{2}\left(1-\mathbf{a}_{2}\right)^{2}-\mathbf{a}_{1}^{2}} \\
& \hat{\mathbf{a}}_{2}=\frac{(1+\mathrm{NSR})\left(\mathbf{a}_{1}^{2}+\mathbf{a}_{2}-\mathbf{a}_{2}^{2}\right)\left(1-\mathbf{a}_{2}\right)-\mathbf{a}_{1}^{2}}{(1+\mathrm{NSR})^{2}\left(1-\mathbf{a}_{2}\right)^{2}-\mathbf{a}_{1}^{2}} .
\end{aligned}
$$

For NSR $>0$ the absolute values of the parameters are underestimated, i.e. $\left|\hat{\mathbf{a}}_{1}\right|<$ $\left|\mathbf{a}_{1}\right|$ and $\left|\hat{\mathbf{a}}_{2}\right|<\left|\mathbf{a}_{2}\right|$, see Appendix B. As an example $\mathbf{a}_{1}=1.7$ and $\mathbf{a}_{2}=-0.8$ are used. In Fig. 2 the parameter estimates are shown as a function of the noiseto-signal ratio. Note that $a_{2}$ first increases and then decreases for increasing noise-to-signal ratio.

In the two dimensional scenario the calculations become more complex. Covariances for a two dimensional $\mathrm{AR}[2]$ process with influence only from $x_{2}$ onto $x_{1}$ at lag one are given in Appendix C. Based on these covariances, the estimated 

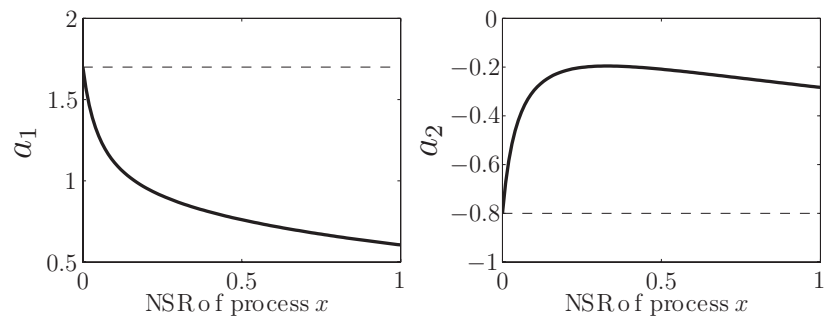

Figure 2: Coefficients of one dimensional AR[2] process estimated in the presence of observational noise.
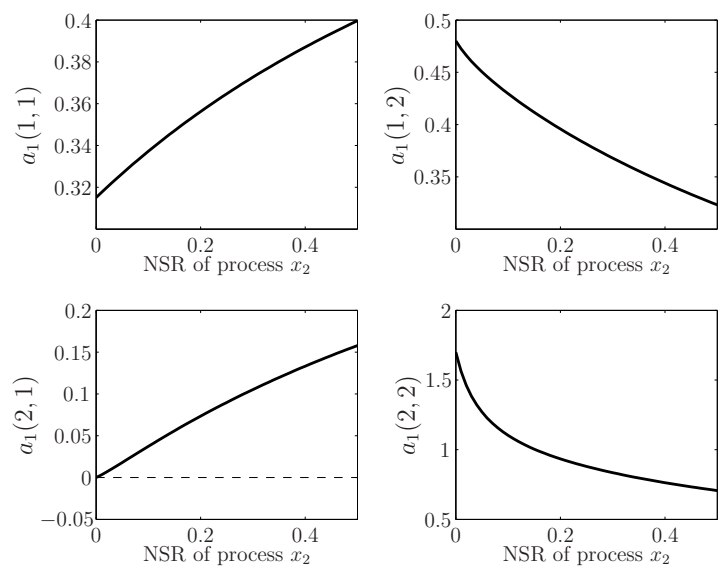

Figure 3: Coefficients $\mathbf{a}_{1}$ of two dimensional AR[2] process with unidirectional influence from process $x_{2}$ to process $x_{1}$ estimated in the presence of observational noise. Noise-to-signal ratio for process $x_{1}$ is 1 .

parameters in dependence on the noise-to-signal ratio can be calculated. As an example $\mathbf{a}_{1}$ and $\mathbf{a}_{2}$ of Eqn. (3) with $c=0.3$ are used. The NSR of process $x_{1}$ is set to one while the dependence on the NSR of $x_{2}$ is investigated. The estimated parameters in dependence on the noise-to-signal ratio are shown in Fig. 3 and 4. Similar to the one dimensional scenario, the coefficients $a_{2}(2,1)$ and $a_{2}(2,2)$ are non-monotone. Note that the absolute value of the estimated parameters can be larger than the absolute value of the true parameters $\left(a_{1}(1,1), a_{1}(2,1)\right.$, and $\left.a_{2}(2,1)\right)$. Since for NSR $>0$ also the estimations of the parameters which are zero in the simulation differ from zero $\left(a_{1}(2,1)\right.$ and $\left.a_{2}(2,1)\right)$, false influences 

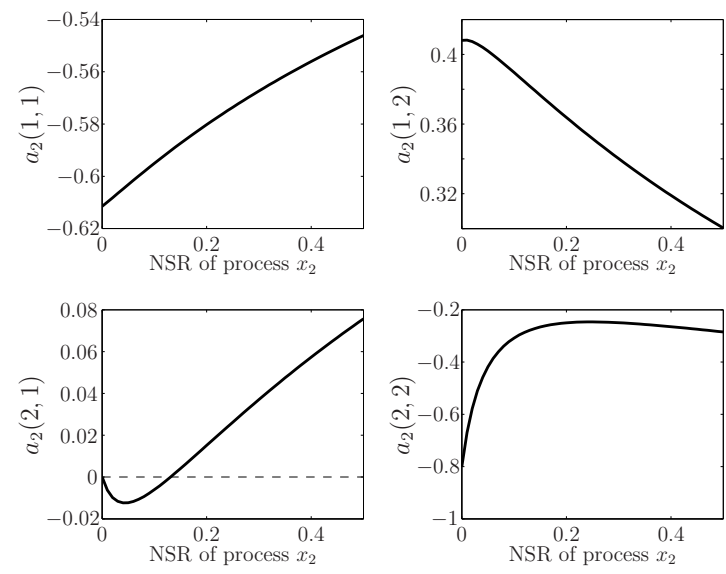

Figure 4: Coefficients $\mathbf{a}_{2}$ of two dimensional AR[2] process with unidirectional influence from process $x_{2}$ to process $x_{1}$ estimated in the presence of observational noise. Noise-to-signal ratio for process $x_{1}$ is 1 .

are estimated for a unidirectional influence.

Renormalised partial directed coherence detects influences if the corresponding parameters for any lag differ from zero. Thus, from the above calculations, it is expected, that an influence from process $x_{1}$ to process $x_{2}$ is falsely detected if process $x_{2}$ is afflicted with observational noise. In Fig. 1 this is indeed observed for low and high noise-to-signal ratios of process $x_{1}$. In-between, there is a region with non-zero noise-to-signal ratio of process $x_{2}$ where correctly but unexpectedly no influence from $x_{1}$ onto $x_{2}$ is detected. Investigating the two corresponding coefficients in more detail reveals that for lag two the coefficient is negative at first and then increases with increasing noise-to-signal ratio. Since the coefficients are estimated, there is a certain confidence band for which the coefficients are compatible with zero when in fact they are non-zero. If the coefficient of lag one is still within this band while the coefficient of lag two becomes compatible with zero, both are compatible with zero and thus, no influence is detected. This is illustrated in Fig. 5(a), where in the shaded region both coefficients are compatible with zero. An increase of the number of data points for the estimation of the coefficients leads to smaller confidence bands. 


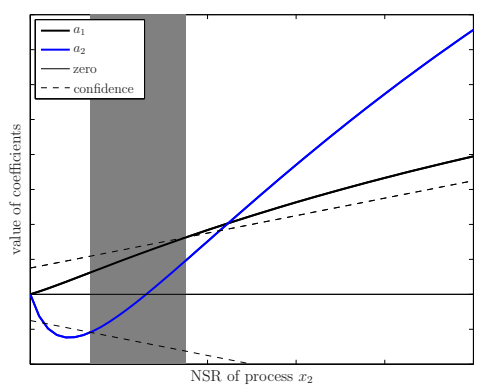

(a)

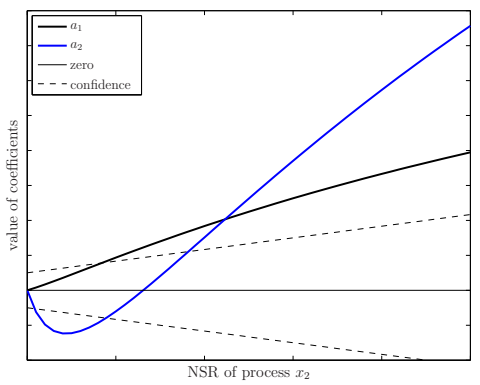

(b)

Figure 5: Schematic of theoretical values of estimated coefficients for the influence from $x_{1}$ onto $x_{2}$ in the presence of observational noise. Noise-to-signal ratio for process $x_{1}$ differs from zero. (a) There is a region, where both coefficients are compatible with zero (shaded region). (b) For an increased number of data points no such region exists.

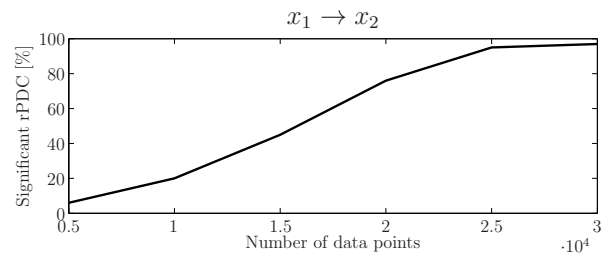

Figure 6: Percentage of realisations with significant renormalised partial directed coherence at the oscillation frequency of the driving process $(0.12 \mathrm{~Hz})$. Noise-to-signal ratio of process $x_{1}$ is set to 1 and NSR $=0.25$ for process $x_{2}$. For the estimation, a model order of $p=2$ is used. In the simulated system, there is no influence from $x_{1}$ onto $x_{2}$. Thus, the detections of this influence are false positive.

This in turn leads to a cancellation of the observed effect. Schematically, the scenario for an increased number of data points is shown in Fig. 5(b). In this case, the region for which no influence is detected has vanished. The detection of the influence from $x_{1}$ onto $x_{2}$ is a false positive detection but expected since observational noise is neglected in the estimation procedure.

Estimation of renormalised partial directed coherence for different numbers of data points supports the considerations above. The noise-to-signal ratio of process $x_{1}$ is set to one and NSR $=0.25$ for process $x_{2}$. The number of data points is varied between $N=5,000$ and $N=30,000$ in steps of 5,000. Results are shown in Fig. 6. As predicted, the percentage of false positive detections 

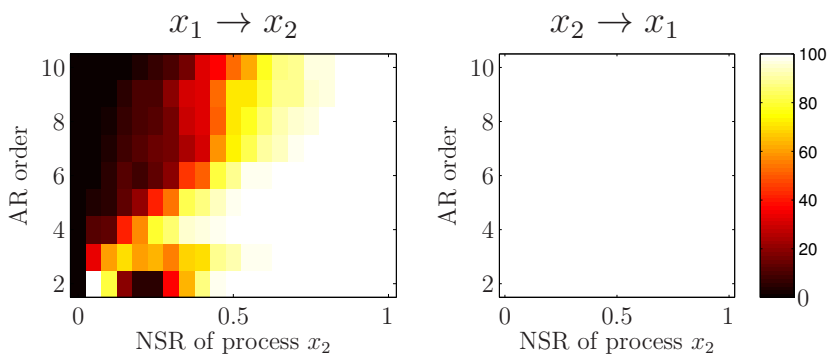

Figure 7: Percentage of realisations with significant renormalised partial directed coherence at the oscillation frequencies of the driving process, 0.12 and $0.05 \mathrm{~Hz}$, respectively. Noise-tosignal ratio of process $x_{1}$ is set to 1 . The model order for estimation is varied between $p=2$ and $p=10$. In the simulated system, there is no influence from $x_{1}$ onto $x_{2}$. Thus, the bright regions are false positive detections of this influence.

increases with increasing $N$. For large $N$, the simulated results, thus, fulfil the theoretical findings in the presence of observational noise.

An increase of the order for estimation leads to a cancellation of the effect explained above since the degree of freedom is increased by the increased number of coefficients that are estimated. Setting the noise-to-signal ratio of process $x_{1}$ to one, the renormalised partial directed coherence is estimated using model orders $p=2$ to $p=10$. Results are shown in Fig. 7 .

Increasing the order of the process yields a better approximation of the true system. On the one hand, using a higher order for the estimation, i.e. $p=10$ (Fig. 8), solves the problem of spuriously detecting the influence from $x_{1}$ onto $x_{2}$ for low noise-to-signal ratios. On the other hand, a higher order requires the estimation of more parameters. Thus, in order to reduce the number of parameters, observational noise should be accounted for in the autoregressive model.

\section{State Space Model}

Linear state space modelling is a powerful framework to estimate processes with observational noise (Harvey, 1994; Kitagawa and Gersch, 1996). To include observational noise explicitly, the state space model representation of a VAR[1] 

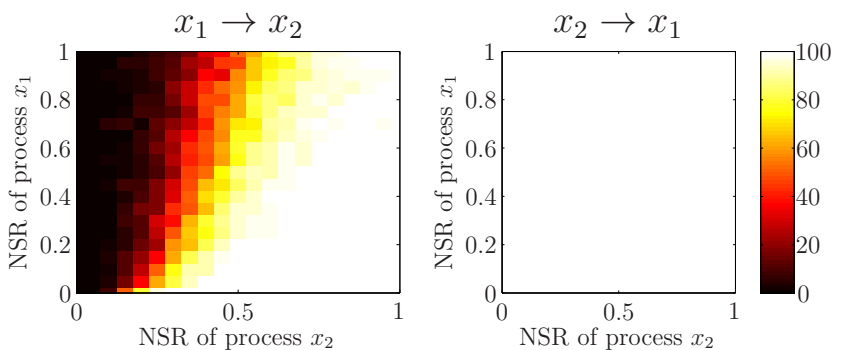

Figure 8: Percentage of realisations with significant renormalised partial directed coherence at the oscillation frequencies of the driving process, 0.12 and $0.05 \mathrm{~Hz}$, respectively. A model order of $p=10$ is used for the estimation. In the simulated system, there is no influence from $x_{1}$ onto $x_{2}$. Thus, the bright regions are false positive detections of this influence.

process (Shumway and Stoffer, 1982; Harvey, 1994)

$$
\begin{array}{rlrl}
\vec{u}(t) & =\mathbf{A} \vec{u}(t-1)+\vec{\varepsilon}_{u}(t) & & \vec{\varepsilon}_{u}(t) \sim \mathcal{N}\left(\overrightarrow{0} \mid \mathbf{Q}_{u}\right) \\
\vec{y}(t) & =\mathbf{C}_{u} \vec{u}(t)+\vec{\eta}(t) & \vec{\eta}(t) \sim \mathcal{N}(\overrightarrow{0} \mid \mathbf{R})
\end{array}
$$

where $\vec{u}(t)$ is the multivariate hidden process and $\vec{y}(t)$ the observed process contaminated with Gaussian noise $\vec{\eta}(t)$, is used.

A stationary VAR process of order $p$ and dimension $n$ (Eq. (1))

$$
\left(\begin{array}{c}
x_{1}(t) \\
x_{2}(t) \\
\vdots \\
x_{n}(t)
\end{array}\right)=\sum_{r=1}^{p} \mathbf{a}(r)\left(\begin{array}{c}
x_{1}(t-r) \\
x_{2}(t-r) \\
\vdots \\
x_{n}(t-r)
\end{array}\right)+\left(\begin{array}{c}
\epsilon_{1}(t) \\
\epsilon_{2}(t) \\
\vdots \\
\epsilon_{n}(t)
\end{array}\right)
$$

can be rewritten as a first order process by augmenting its dimension. Therefore, all past information needed to predict $\vec{x}(t)$ is collected in one single new $n_{u^{-}}$ dimensional vector

$$
\vec{u}(t-1)=\left(\vec{x}^{T}(t-1), \vec{x}^{T}(t-2), \ldots, \vec{x}^{T}(t-p)\right)^{T},
$$


with dimension $n_{u}=n p$. The model equation

$$
\underbrace{\left(\begin{array}{c}
\vec{x}(t) \\
\vec{x}(t-1) \\
\vdots \\
\vec{x}(t-p+1)
\end{array}\right)}_{\vec{u}(t)}=\underbrace{\left(\begin{array}{cccc}
\mathbf{a}_{1} & \mathbf{a}_{2} & \cdots & \mathbf{a}_{p} \\
\mathbf{I}_{n} & \mathbf{0}_{n} & \cdots & \mathbf{0}_{n} \\
\vdots & \ddots & \ddots & \vdots \\
\mathbf{0}_{n} & \cdots & \mathbf{I}_{n} & \mathbf{0}_{n}
\end{array}\right)}_{\mathbf{A}} \underbrace{\left(\begin{array}{c}
\vec{x}(t-1) \\
\vec{x}(t-2) \\
\vdots \\
\vec{x}(t-p)
\end{array}\right)}_{\vec{u}(t-1)}+\underbrace{\left(\begin{array}{c}
\vec{\varepsilon}_{x}(t) \\
\overrightarrow{0} \\
\vdots \\
\overrightarrow{0}
\end{array}\right)}_{\vec{\varepsilon}_{u}(t)}
$$

with

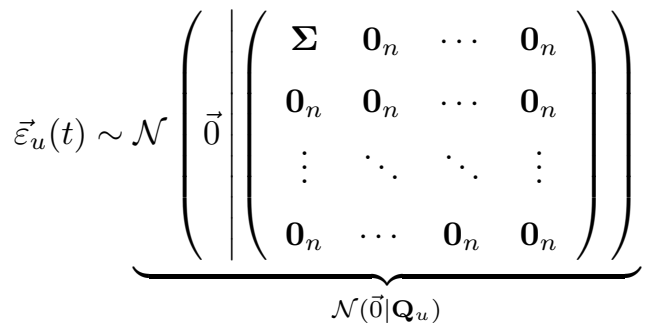

of the new vector $\vec{u}(t)$ is an equivalent representation of the $\operatorname{VAR}[p]$ process $\vec{x}(t)$. The matrices $\mathbf{I}_{n}$ and $\mathbf{0}_{n}$ denote the $n \times n$ identity and the $n \times n$ matrix of zeros. The new representation in Eq. (24) of the $\operatorname{VAR}[p]$ process ensures that the linear state space model of Eqs. (20) and (21) can directly be applied also to $\operatorname{VAR}[p]$ process.

The state space model divides the VAR process afflicted with observational noise into two equations. The first, the system equation (20) or (24), describes the $\operatorname{VAR}[p]$ process. The second, the observation equation (21) or

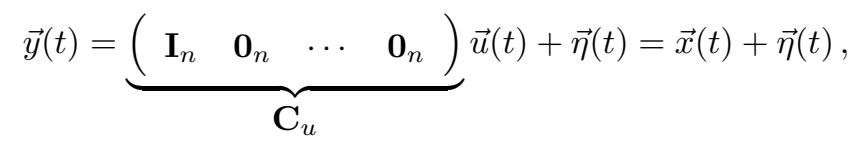

describes the observation of the $n$-dimensional vector $\vec{x}(t)$ of the $\operatorname{VAR}[p]$ process with additive observational noise $\vec{\eta}_{t}$ of dimension $n$.

\subsection{Estimation of Parameters}

In linear state space models, the optimal estimators for the hidden process $\vec{u}(t)$, given certain observations $\{\vec{y}(1), \ldots, \vec{y}(s)\}$ and assuming knowledge about the true parameters, are given by the Kalman filter (Kalman, 1960; 
Kalman and Bucy, 1961) and smoother (Rauch et al., 1965; Ansley and Kohn, 1982). The Kalman filter yields the conditional expectation value $\vec{u}(t \mid t):=$ $\langle\vec{u}(t) \mid \vec{y}(1), \ldots, \vec{y}(t)\rangle$ considering only observations up to time $t$ and thus can be applied on-line. The Kalman smoother calculates the conditional expectation value of the hidden process $\vec{u}(t \mid N):=\langle\vec{u}(t) \mid \vec{y}(1), \ldots, \vec{y}(N)\rangle$ by taking all $N$ observations into account. These two estimators rely on the knowledge of the parameters, i.e. the matrices $\mathbf{A}, \mathbf{Q}_{u}, \mathbf{C}, \mathbf{R}$. The Expectation-Maximisation (EM) algorithm (Dempster et al., 1977) applied to linear Gaussian state space models (Shumway and Stoffer, 1982) presents an iterative algorithm for Maximum Likelihood parameter estimation based on the Kalman filter and smoother. The EM algorithm consists of two steps. In the expectation (E) step, the expectation of the complete data likelihood is calculated given the parameters that were estimated in the previous iteration. Here, the Kalman filter or smoother can be used. The maximisation (M) step then yields parameter updates based on the likelihood estimated in step E. Details can be found in (Shumway and Stoffer, 2000).

The EM algorithm for the linear state space model in combination with the Kalman filter or smoother estimates the $n^{2}(p+2)$ entries of the matrices $\left\{\mathbf{a}_{1}, \ldots, \mathbf{a}_{p}, \boldsymbol{\Sigma}, \mathbf{R}\right\}$ as well as the hidden trajectory $\{\vec{x}(1), \ldots, \vec{x}(N)\}$ using only the observations $\{\vec{y}(1), \ldots, \vec{y}(N)\}$ (Shumway and Stoffer, 1982).

\subsection{Estimation of $r P D C$}

In order to estimate the renormalised partial directed coherence (Eqn. (A.4))

$$
\lambda_{i j}(\omega)=\mathbf{X}_{i j}(\omega)^{\prime} \mathbf{V}_{i j}^{-1}(\omega) \mathbf{X}_{i j}(\omega)
$$

with

$$
\mathbf{V}_{i j}(\omega)=\sum_{l, m=1}^{p} \operatorname{cov}\left(\left(\hat{\mathbf{a}}_{l}\right)_{i j},\left(\hat{\mathbf{a}}_{m}\right)_{i j}\right)\left(\begin{array}{ll}
\cos (l \omega) \cos (m \omega) & \cos (l \omega) \sin (m \omega) \\
\sin (l \omega) \cos (m \omega) & \sin (l \omega) \sin (m \omega)
\end{array}\right)
$$

in addition to the coefficient matrices $\left\{\mathbf{a}_{1}, \ldots, \mathbf{a}_{p}\right\}$ their covariances need to be determined. Thus, the covariances $\operatorname{cov}\left(\left(\mathbf{a}_{l}\right)_{i j},\left(\mathbf{a}_{m}\right)_{i j}\right)$ have to be calculated. 
Here, we present two approaches to address this challenge. First, the covariances $\operatorname{cov}\left(\left(\mathbf{a}_{l}\right)_{i j},\left(\mathbf{a}_{m}\right)_{i j}\right)$ are calculated using the incomplete data likelihood (Shumway and Stoffer, 2000)

$$
-\ln \mathrm{L}_{\vec{y}}(\boldsymbol{\Theta})=\frac{1}{2} \sum_{t=1}^{N} \log |\Sigma(t, \boldsymbol{\Theta})|+\frac{1}{2} \sum_{t=1}^{N} \epsilon(t, \boldsymbol{\Theta})^{T} \Sigma(t, \boldsymbol{\Theta})^{-1} \epsilon(t, \boldsymbol{\Theta})
$$

with innovations

$$
\begin{aligned}
\epsilon(t, \boldsymbol{\Theta}) & =\vec{y}(t)-\mathbf{C} \vec{u}(t \mid t-1) \\
\Sigma(t, \boldsymbol{\Theta}) & =\mathbf{C P}(t \mid t-1) \mathbf{C}^{T}+\mathbf{R}
\end{aligned}
$$

and

$$
\begin{aligned}
\mathbf{P}(t \mid t-1) & =\mathbf{A} \mathbf{P}(t-1 \mid t-1) \mathbf{A}^{T}+\mathbf{Q} \\
\mathbf{K}(t) & =\mathbf{P}(t \mid t-1) \mathbf{C}^{T}\left(\mathbf{C P}(t \mid t-1) \mathbf{C}^{T}+\mathbf{R}\right)^{-1} \\
\mathbf{P}(t \mid t) & =(1-\mathbf{K}(t) \mathbf{C}) \mathbf{P}(t \mid t-1) \\
\vec{u}(t \mid t-1) & =\mathbf{A} \vec{u}(t-1 \mid t-1) \\
\vec{u}(t \mid t) & =\vec{u}(t \mid t-1)+\mathbf{K}(t)(\vec{y}(t)-\mathbf{C} \vec{u}(t \mid t-1))
\end{aligned}
$$

from the Kalman filter. The covariances can be calculated by the inverse of the Hessian of $-\ln \mathrm{L}_{\vec{y}}(\boldsymbol{\Theta})$ (Shumway and Stoffer, 2000). Thus, the second derivatives of the likelihood have to be derived. This can be done analytically as shown in Appendix D. The second derivatives of all parameters, i.e. all entries of $\mathbf{A}, \mathbf{Q}$ and $\mathbf{R}$, have to be calculated. They are then arranged in a matrix. Inverting this matrix yields the covariances.

Second, an approach based on parametric bootstrap realisations (Efron and Tibshirani, 1998) yields the desired covariances. Using the EM-algorithm, an estimate of the parameters is achieved for the time series under investigation. The estimated parameters are then used to create parametric bootstrap realisations of the process by simulating the model with these parameters. For all bootstrap realisations, parameters are then estimated. The empirical covariances of the parameter estimates of the bootstrap are then used as $\operatorname{cov}\left(\left(\mathbf{a}_{l}\right)_{i j},\left(\mathbf{a}_{m}\right)_{i j}\right)$. 


\subsection{Simulations}

In this section, the parameters estimated based on the state space model are used to calculate the renormalised partial directed coherence (Eq. (A.4)). The covariances $\operatorname{cov}\left(\left(\mathbf{a}_{l}\right)_{i j},\left(\mathbf{a}_{m}\right)_{i j}\right)$ needed, are estimated first, based on a bootstrap approach and second, by analytical differentiation of the likelihood function. Thus, observational noise is accounted for in the model and renormalised partial directed coherence can be estimated for the underlying process.

We estimate rPDC based on the state space model using the same simulated data as in Sec. 2. Here, the noise-to-signal ratio for both processes is the same. For each noise-to-signal ratio and each of the 100 realisations, the renormalised partial directed coherence is estimated using the two different approaches. Figure 9 shows exemplary time series including simulated time series (yellow), time series contaminated with observational noise (black), and the denoised time series (red). It can be seen that using the state space model the original time series can be approximated very well. Additionally, the respective spectra are shown. From the noisy spectra it is obvious that without the state space model the true spectra cannot be approximated at all.

Results for $\mathrm{rPDC}$ using $p=2$ and covariances obtained from analytical differentiation of the likelihood are shown in Fig. 10. In this case, around 5\% false positive conclusions occurred in accordance with the 5\%-significance level.

Results for for $\mathrm{rPDC}$ using $p=2$ and covariances obtained from 100 bootstrap realisations are similar (not shown). Again, around 5\% false positive conclusions occurred at a 5\%-significance level. Thus, using the state space model, it is possible to correctly reveal the interaction structure even in the presence of observational noise.

\subsection{Strengths of the interactions}

To investigate the implications for the strengths of the interactions, we var-

ied the parameter $c$ in the model system Eq. (1) between 0 and 0.5 in steps of 0.01. In the first simulation study, we kept the noise-to-signal ratio constant at 

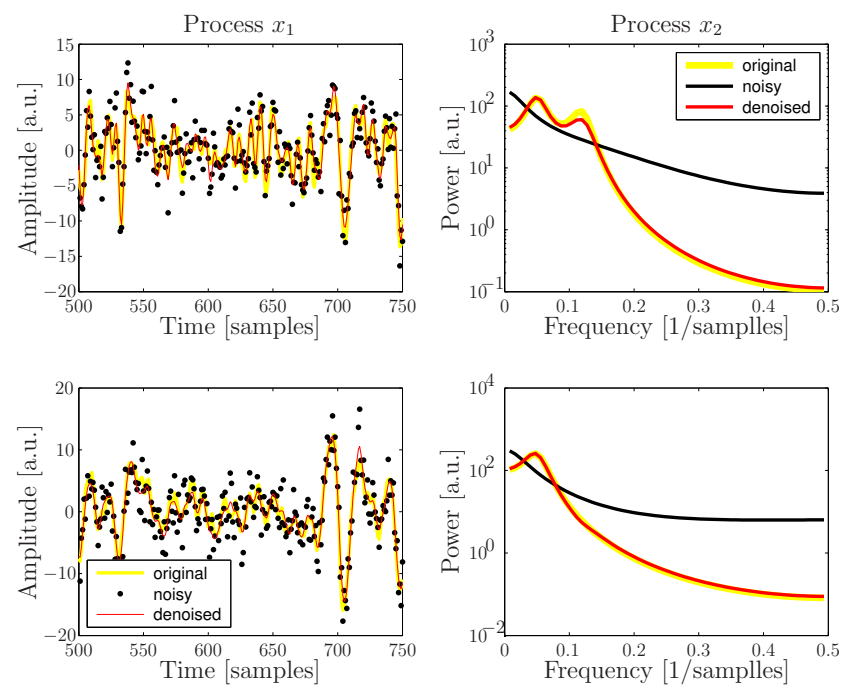

Figure 9: Exemplary time series (left) and spectra (right) of simulated two dimension AR[2] with NSR $=0.25$. Original (yellow) depicts simulated data before observational noise is added. Noisy (black) refers to results without state space model. Denoised (red) shows results of estimation using the state space model. A model order of $p=2$ is used in both cases.
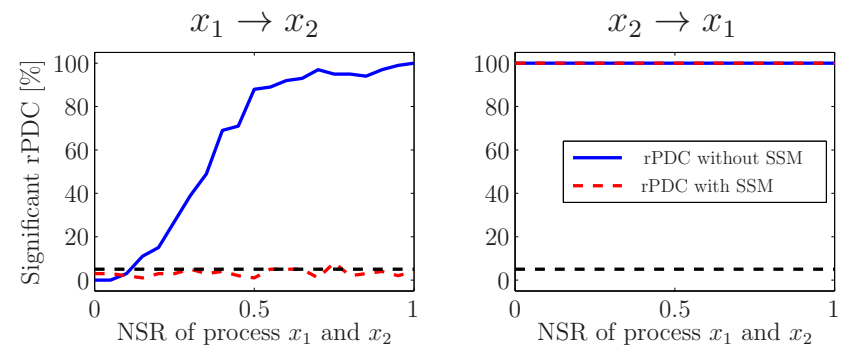

Figure 10: Percentage of realisations with significant renormalised partial directed coherence at the oscillation frequencies of the driving process, 0.12 and $0.05 \mathrm{~Hz}$, respectively. Shown in red, estimation using the state space model with a model order of $p=2$ and covariances obtained from analytical differentiation of the likelihood. Blue lines refer to results without state space model and $p=10$ (diagonal of Fig. 8). 

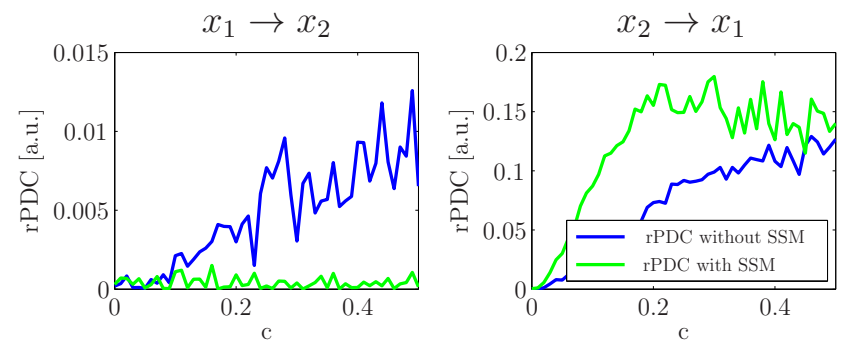

Figure 11: rPDC estimated naively (blue curves) and based on the EM algorithm with analytical covariance (green curves). Noise-to-signal ratio is constant.

$\mathrm{NSR}=0.5$. The results for the rPDC estimated based on the naive application of the Yule-Walker equations and the EM based estimation with the analytically derived covariance matrix is depicted in Fig. 11. It becomes evident again that the naive estimation, blue curves, lead to spurious Granger-causalities, while the EM based estimation, green curves, correctly reveals the true interaction structure. Note that for the influence from $x_{1}$ onto $x_{2}$ (Fig. 11 left) the rPDC values of the EM based estimation do not increase with increasing coupling strengths. For the opposite direction, $x_{2}$ onto $x_{1}$, which is present in the simulation, rPDC values increase with increasing coupling strengths. The increase is steeper for the EM based estimation. Thus, present interactions are detected for a lower coupling strength in this case. For higher coupling strengths the curve indicating the correct interaction saturates for the EM based estimation. This, at a first glance, might be counter-intuitive since it does not represent the increase in the coupling parameter $c$. It can be explained as follows.

The noise-to-signal ratio was kept constant (NSR $=0.5)$, in other words, the noise contribution depends on the coupling strength. For increasing coupling strength the parameters are still estimated correctly, see Fig. 12, but the standard deviations of the parameter estimates increase with increasing coupling strength (red curves). As the standard deviations quadratically enter the covariance matrix, which in turn enters the estimator for the $\mathrm{PPDC}$, the saturation effect can be understood as being related to the dependence of the observational noise contribution on the coupling strength. 


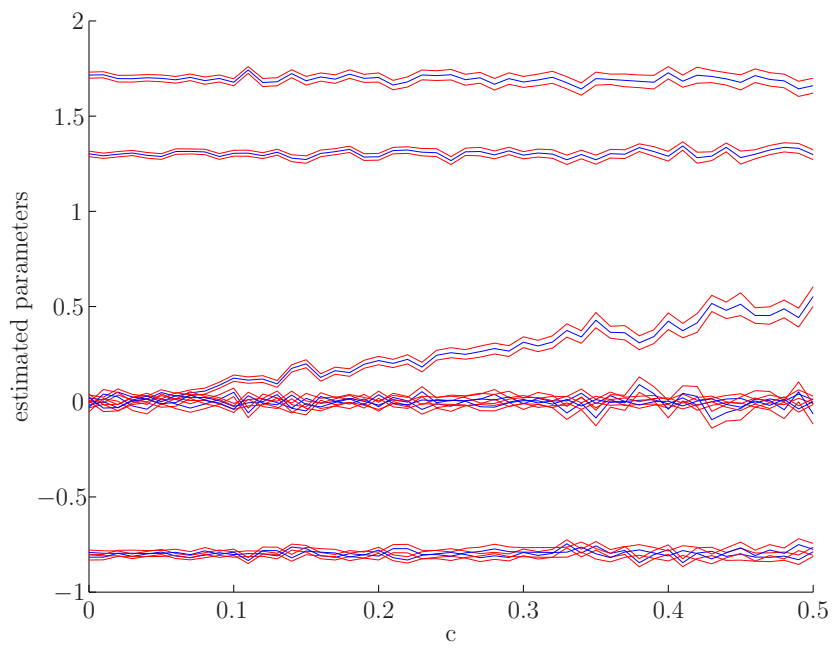

Figure 12: Estimated parameters and their standard deviations for all 8 parameters of model system Eq. 1. The parameter $c$ (third line from top) gradually differs from zero in accordance with the simulation.

As this dependence is counter-intuitive for applications, we simulated the same scenario with constant variance of the observational noise. We used a standard deviation of the observational noise of $\sigma=5$. This corresponds to $\mathrm{NSR}=1$ for process $x_{2}$ and NSR of process $x_{1}$ decreasing from 4 to 0.5 for increasing interaction strength. Despite higher fluctuations for larger coupling strength, the rPDC quantifies the strengths of the interactions as already shown in (Schelter et al., 2009) for the noise free case (Fig. 13). We emphasise again that a naive application of rPDC without using the analytic statistics and the EM algorithm would have resulted in spurious interactions between the processes, independent of the chosen model order.

\subsection{Application to mice EEG}

In an exemplary application, we analyse electroencephalogram (EEG) data of mice. EEG recordings were obtained under freely-moving conditions with a wireless device. The signal was sampled at $199 \mathrm{~Hz}$. Three electrodes were placed into the skull above prefrontal cortex $(\mathrm{PFx})$ and bilaterally above the hippocampus (left: lHC, right: $\mathrm{rHC}$ ), respectively. For details of the recording 


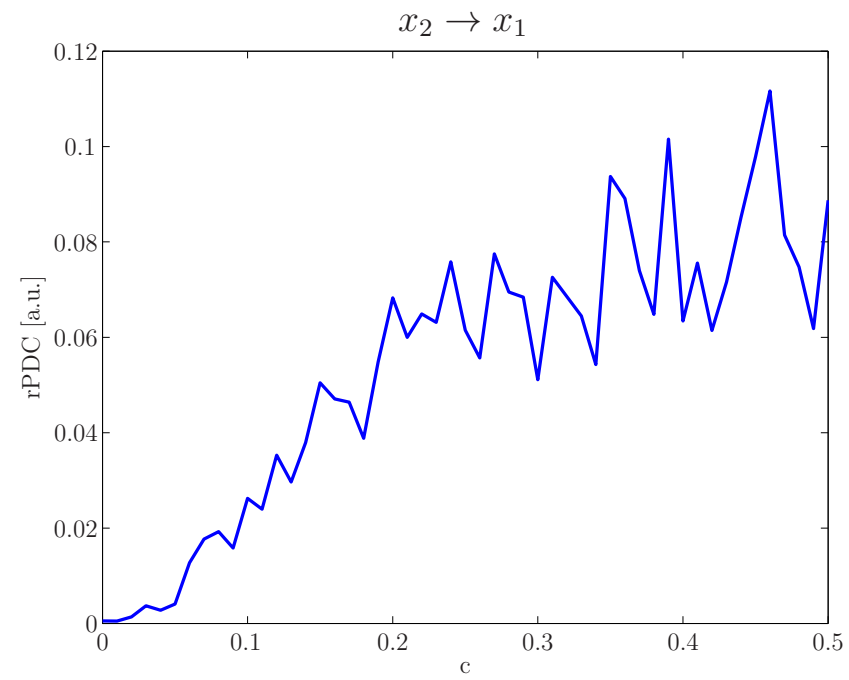

Figure 13: rPDC estimated based on the EM algorithm using the analytical covariance (blue curve). Variance of the observational noise is constant.

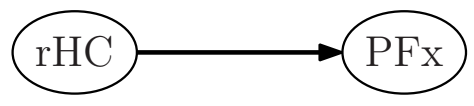

(a)

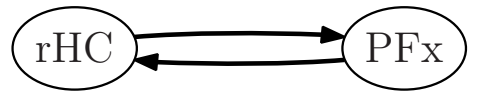

(b)

Figure 14: Results for the analysis of EEG data from five mice. Interaction structure as revealed using $\mathrm{rPDC}$ estimated based on the EM algorithm. Only significant influences are shown. (a) results for mice 1,2 and 3 (b) results for mice 4 and 5.

technique, see (Jyoti et al., 2010). Data were downsampled to $40 \mathrm{~Hz}$ and a model order of $p=2$ was used in the state space model. We analysed one segment of 100 seconds of $\mathrm{rHC}$ and PFx in the quiet wake state for each of five mice. The interaction structure that was revealed is shown in Fig. 14. We found a statistically significant unidirectional influence from $\mathrm{rHC}$ to PFx in three mice and a statistically significant bidirectional influence in the remaining two mice. Thus, $60 \%$ of the investigated mice showed a unidirectional influence from $\mathrm{rHC}$ to PFx in the quiet wake state.

EEG signals have several frequency contents that are associated with different tasks. When the prefrontal cortex is involved, theta band oscillations have 
been shown to mediate interactions (Anderson et al., 2010). In this study we examined only the presence of influences. A detailed analysis of the different frequency bands requires the use of a higher model order and will be subject to a subsequent, more applied publication.

The recorded EEG electrodes share a common reference. In the past, a common reference has been shown to potentially cause spurious detections of Granger causality (Bollimunta et al., 2009). It remains to be investigated how the use of a state space model as presented here affects this finding. We mention that it is in principle possible to account for instantaneous interactions such as those expected to occur when using common referencing in state space modelling by adjusting the observation matrix. Exploiting this is beyond the scope of this manuscript.

\section{Conclusion}

In this manuscript, we investigate the influence of observational noise on the detection of interactions. We showed that direct application of renormalised partial directed coherence leads to false positive conclusions. As this is intrinsic to the estimation of the parameters of the vector autoregressive model, similar problems would arise for any Granger-causality measure that directly relies on VAR models.

For that reason, state space modelling as a means to deal with observational noise was presented. State-space modelling explicitly includes observational noise in the model and thus allows to estimate parameters without bias. Renormalised partial directed coherence was then calculated from the parameters estimated in the state space model. Importantly, covariances of the coefficients were calculated both based on a bootstrap based approach and an analytical differentiation of the likelihood. We emphasise that only based on the reliable derivation of the covariances a trustworthy statistical inference is possible, avoiding false positive conclusions about the interaction structure. Repeating the simulation with both versions of this advanced method reveals the true interaction structure, including a reasonable approximation for the strengths of 
the interactions. An application to mice EEG showed the potential of the presented approach. In future studies we will investigate different vigilance states and their potentially different network structures.

Here, we focused on linear Granger-causality for stationary processes. Extensions for non-stationary as well as non-linear processes are available (e.g. Omidvarnia et al., 2013; Li et al., 2012; Sommerlade et al., 2012; Chen et al., 2004; Schelter et al., 2014). Some of these extensions are able to deal with observational noise for others the influence of observational noise needs to be investigated in future studies (Omidvarnia et al., 2013; Li et al., 2012).

For the examples presented here, we assumed that the observational noise is uncorrelated Gaussian white noise, which is often a good first approximation. However, the framework does allow other assumptions such as correlations or coloured noise to be included in the model. To this aim the observation equation (Eq. (21)) as well as the Kalman filter filter have to be modified by, e.g., augmenting the state space model to include a dynamic equation for the noise. This will have also an impact on the derivation of the critical values for a given significance level as the derivatives will need to be adjusted. Discussing this in more detail is beyond the scope if this manuscript.

In summary, a naive application of renormalised partial directed coherence and any other Granger-causality measure based on vector autoregressive models should be avoided. Instead, state space model based estimations should be used in order to avoid false positive conclusions. The analytical derivation of the statistics presented in this manuscript enables a rigorous evaluation of the results. Noteworthy, the statistics can be readily applied to other measures for Granger-causality as well.

\section{Acknowledgements}

This work was supported by the German Science Foundation (Ti315/42), the German Federal Ministry of Education and Research (BMBF grant 01GQ0420), and the Excellence Initiative of the German Federal and State 
Governments. B.S. is indebted to the Kosterlitz centre for the financial support of this research project.

\section{References}

Anderson, K. L., Rajagovindan, R., Ghacibeh, G. A., Meador, K. J., Ding, M., 2010. Theta oscillations mediate interaction between prefrontal cortex and medial temporal lobe in human memory. Cereb. Cortex 20, 1604-12.

Ansley, C. F., Kohn, R., 1982. A geometrical derivation of the fixed interval smoothing algorithm. Biometrika 69, 486-7.

Arnhold, J., Grassberger, P., Lehnertz, K., Elger, C. E., 1999. A robust method for detecting interdependencies: Application to intracranially recorded EEGs. Physica D 134, 419-30.

Arnold, M., Miltner, W. H. R., Witte, H., Bauer, R., Braun, C., 1998. Adaptive AR modeling of nonstationary time series by means of Kalman filtering. IEEE T. Bio-Med. Eng. 45, 553-62.

Baccalá, L. A., Sameshima, K., 2001. Partial directed coherence: A new concept in neural structure determination. Biol. Cybern. 84, 463-74.

Bahraminasab, A., Ghasemi, F., Stefanovska, A., McClintock, P. V. E., Friedrich, R., 2009. Physics of brain dynamics: Fokker-Planck analysis reveals changes in EEG delta and theta activity during anaesthesia. New J. Phys. 11, 103051.

Bollimunta, A., Chen, Y., Schroeder, C. E., Ding, M., 2009. Characterizing Oscillatory Cortical Networks with Granger Causality. In: Rubin, J., Josic, K., Matias, M., Romo, R. (Eds.), Coherent Behavior in Neuronal Networks. Springer, New York, pp. 169-89.

Bowers, E. J., Murray, A., 2004. Interaction between cardiac beat-to-beat interval changes and systolic blood pressure changes. Clin. Autonom. Res. 14, $92-8$. 
Brillinger, D. R., 1981. Time Series: Data Analysis and Theory. Holden-Day, San Francisco.

Brockwell, P. J., Davis, R. A., 1998. Time Series: Theory and Methods. Springer, New York.

Chen, Y., Rangarajan, G., Feng, J., Ding, M., 2004. Analyzing multiple nonlinear time series with extended Granger causality. Phys. Lett. A 324, 26-35.

Chen, W. Y., Wasterlain, C. G., 2006. Status epilepticus: Pathophysiology and management in adults. Lancet Neurol. 5, 246-56.

Chicharro, D., Andrzejak, R. G., 2009. Reliable detection of directional couplings using rank statistics. Phys. Rev. E 80, 026217.

Dahlhaus, R., 2000. Graphical interaction models for multivariate time series. Metrika 51, 157-72.

Dahlhaus, R., Eichler, M., 2003. Causality and graphical models for time series. In: Green, P., Hjort, N., Richardson, S. (Eds.), Highly Structured Stochastic Systems. Oxford University Press, pp. 115-37.

Dempster, A. P., Laird, N. M., Rubin, D. B., 1977. Maximum likelihood from incomplete data via EM algorithm. J. Roy. Stat. Soc. 39, 1-38.

Dhamala, M., Rangarajan, G., Ding, M., 2008. Estimating Granger causality from Fourier and wavelet transforms of time series data. Phys. Rev. Lett. 100, 018701.

Efron, B., Tibshirani, R. J., 1998. An Introduction to the Bootstrap. Chapman \& Hall, New York.

Eichler, M., 2000. Markov properties for graphical time series models. Preprint University of Heidelberg.

Eichler, M., 2006. Graphical modeling of dynamic relationships in multivariate time series. In: Schelter, B., Winterhalder, M., Timmer, J. (Eds.), Handbook of Time Series Analysis. Wiley-VCH, Ch. 14, pp. 335-72. 
Frenzel, S., Pompe, B., 2007. Partial mutual information for coupling analysis of multivariate time series. Phys. Rev. Lett. 99, 204101.

Geweke, J., 1982. Measurement of linear dependence and feedback between multiple time series. J. Am. Stat. Assoc. 77, 304-13.

Geweke, J., 1984. Measures of conditional linear dependence and feedback between time series. J. Am. Stat. Assoc. 79, 907-15.

Granger, J., 1969. Investigating causal relations by econometric models and cross-spectral methods. Econometrica 37, 424-38.

Halliday, D. M., Rosenberg, J. R., 2000. On the application and estimation and interpretation of coherence and pooled coherence. J. Neurosci. Meth. 100, $173-4$.

Harvey, A. C., 1994. Forecasting Structural Time Series Models and the Kalman Filter. Cambridge University Press, Cambridge.

Hesse, W., Möller, E., Arnold, M., Schack, B., 2003. The use of time-variant EEG Granger causality for inspecting directed interdependencies of neural assemblies. J. Neurosci. Meth. 124, 27-44.

Jyoti, A., Plano, A., Riedel, G., Platt, B., 2010. EEG, activity, and sleep architecture in a transgenic $\mathrm{A} \beta \mathrm{PP}_{\mathrm{swe}} / \mathrm{PSEN1}_{\mathrm{A} 246 \mathrm{E}}$ Alzheimer's disease mouse. J. Alzheimers Dis. 22, 873-87.

Kalman, R. E., 1960. A new approach to linear filtering and prediction problems. J. Basic Eng.-T. ASME 82, 35-46.

Kalman, R. E., Bucy, R. S., 1961. New results in linear filtering and prediction theory. J. Basic Eng.-T. ASME 83, 95-107.

Kamiński, M. J., Blinowska, K. J., 1991. A new method of the description of the information flow in the brain structures. Biol. Cybern. 65, 203-10. 
Kamiński, M. J., Blinowska, K. J., Szelenberger, W., 1997. Topographic analysis of coherence and propagation of EEG activity during sleep and wakefulness. Electroenceph. Clin. Neurophys. 102, 216-27.

Keyl, C., Dambacher, M., Schneider, A., Passino, C., Wegenhorst, U., Bernardi, L., 2000. Cardiocirculatory coupling during sinusoidal baroreceptor stimulation and fixed-frequency breathing. Clinical Science 99, 113-24.

Kitagawa, G., Gersch, W., 1996. Smoothness Priors Analysis of Time Series. No. 116 in Lecture Notes in Statistics. Springer, New York.

Korzeniewska, A., Kasicki, S., Kamiński, M. J., Blinowska, K. J., 1997. Information flow between hippocampus and related structures during various types of rat's behavior. J. Neurosci. Meth. 73, 49-60.

Li, Y., Wei, H.-L., Billings, S. A., Liao, X.-F., 2012. Time-varying linear and nonlinear parametric model for Granger causality analysis. Phys. Rev. E 85, 041906 .

Lütkepohl, H., 1993. Introduction to Multiple Time Series Analysis. Springer.

Nalatore, H., Ding, M., Rangarajan, G., 2007.Mitigating the effects of measurement noise on Granger causality. Phys. Rev. E 75, 031123.

Nalatore, H., Ding, M., Rangarajan, G., 2009.Denoising neural data with statespace smoothing: method and application. J. Neurosci. Meth. 179, 131-41.

Newbold, P., 1978.Feedback Induced by Measurement Errors. Int. Econ. Rev. $19,787-91$.

Nicolelis, M. A. L., Fanselow, E. E., 2002. Thalamocortical optimization of tactile processing according to behavioral state. Nat. Neurosci. 5, 517-23.

Nollo, G., Faes, L., Porta, A., Antolini, R., Ravelli, F., 2005. Exploring directionality in spontaneous heart period and systolic pressure variability interactions in humans: Implications in the evaluation of baroreflex gain. Am. J. Physiol. Heart. Circ. Physiol. 288, 1777-85. 
Nolte, G., Ziehe, A., Nikulin, V. V., Schlögl, A., Krämer, N., Brismar, T., Müller, K.-R., 2008. Robustly estimating the flow direction of information in complex physical systems. Phys. Rev. Lett. 100, 234101.

Omidvarnia, A., Azemi, G., Boashash, B., O’Toole, J. M., Colditz, P. B., Vanhatalo, S., 2013. Measuring time-varying information flow in scalp EEG signals: orthogonalized partial directed coherence. IEEE Trans. Biomed. Eng. 61, 680-93.

Paluš, M., Stefanovska, A., 2003. Direction of coupling from phases of interacting oscillators: An information-theoretic approach. Phys. Rev. E 67, 055201(R).

Paluš, M., Vejmelka, M., 2007. Directionality of coupling from bivariate time series: How to avoid false causalities and missed connections. Phys. Rev. E 75,056211 .

Pitzalis, M. V., Mastropasqua, F., Massari, F., Passantino, A., Colombo, R., Mannarini, A., Forleo, C., Rizzon, P., 1998. Effect of respiratory rate on the relationships between RR interval and systolic blood pressure fluctuations: A frequency-dependent phenomenon. Cardiovasc. Res. 38, 332-9.

Pompe, B., Blidh, P., Hoyer, D., Eiselt, M., 1998. Using mutual information to measure coupling in the cardiorespiratory system. IEEE Eng. Med. Biol. Mag. 17, 32-9.

Prusseit, J., Lehnertz, K., 2008. Measuring interdependences in dissipative dynamical systems with estimated Fokker-Planck coefficients. Phys. Rev. E 77, 041914.

Rauch, H. E., Tung, F., Striebel, C. R., 1965. Maximum Likelihood estimates of linear dynamic systems. AIAA Journal 3, 1445-50.

Romano, M. C., Thiel, M., Kurths, J., Grebogi, C., 2007. Estimation of the direction of the coupling by conditional probabilities of recurrence. Phys. Rev. E 76, 036211. 
Rosenblum, M. G., Cimponeriu, L., Bezerianos, A., Patzak, A., Mrowka, R., 2002. Identification of coupling direction: Application to cardiorespiratory interaction. Phys. Rev. E 65, 041909.

Rosenblum, M. G., Pikovsky, A. S., 2001. Detecting direction of coupling in interacting oscillators. Phys. Rev. E 64, 045202(R).

Sameshima, K., Baccalá, L. A., 1999. Using partial directed coherence to describe neuronal ensemble interactions. J. Neurosci. Meth. 94, 93-103.

Schack, B., Grießbach, G., Arnold, M., Bolten, J., 1995. Dynamic cross spectral analysis of biological signals by means of bivariate ARMA processes with time-dependent coefficients. Med. \& Biol. Eng. \& Comput. 33, 605-10.

Schad, A., Nawrath, J., Jachan, M., Henschel, K., Spindeler, L., Timmer, J., Schelter, B., 2009. Approaches to the detection of direct directed interactions in neuronal networks. In: Velazquez, J. L. P., Wennberg, R. (Eds.), Coordinated Activity in the Brain. Springer, Ch. 3, pp. 43-64.

Schelter, B., Timmer, J., Eichler, M., 2009. Assessing the strength of directed influences among neural signals using renormalized partial directed coherence. J. Neurosci. Meth. 179, 121-30.

Schelter, B., Winterhalder, M., Timmer, J. (Eds.), 2006. Handbook of Time Series Analysis. Wiley-VCH, Berlin.

Schelter, B., Mader, M., Mader, W., Sommerlade, L., Platt, B., Lai, Y.-C., Grebogi, C., Thiel, M., 2014. Overarching framework for data-based modelling. EPL 105, 30004.

Schreiber, T., 2000. Measuring information transfer. Phys. Rev. Lett. 85, 461-4.

Shumway, R. H., Stoffer, D. S., 1982. An approach to time series smoothing and forecasting using the EM algorithm. J. Time Ser. Anal. 3, 253-64.

Shumway, R. H., Stoffer, D. S., 2000. Time Series Analysis and Its Application. Springer, New York. 
Sommerlade, L., Thiel, M., Platt, B., Plano, A., Riedel, G., Grebogi, C., Timmer, J., Schelter, B., 2012. Inference of Granger causal time-dependent influences in noisy multivariate time series. J. Neurosci. Meth. 203, 173-85.

Staniek, M., Lehnertz, K., 2008. Symbolic transfer entropy. Phys. Rev. Lett. $100,158101$.

Strogatz, S. H., Mar 2001. Exploring complex networks. Nature 410 (6825), $268-76$.

Tass, P., Rosenblum, M. G., Weule, J., Kurths, J., Pikovsky, A. S., Volkmann, J., Schnitzler, A., Freund, H. J., 1998. Detection of $n: m$ phase locking from noisy data: Application to magnetoencephalography. Phys. Rev. Lett. 81, $3291-5$.

Timmer, J., 1998. Modeling noisy time series: Physiological tremor. Int. J. Bif. Chaos 8, 1505-16.

Vejmelka, M., Paluš, M., 2008. Inferring the directionality of coupling with conditional mutual information. Phys. Rev. E 77, 026214.

Winterhalder, M., Schelter, B., Hesse, W., Schwab, K., Leistritz, L., Klan, D., Bauer, R., Timmer, J., Witte, H., 2005. Comparison of linear signal processing techniques to infer directed interactions in multivariate neural systems. Sig. Proc. 85, 2137-60.

\section{Appendix A. Renormalised Partial Directed Coherence}

In order to interpret the strengths of influences at different frequencies, renormalised partial directed coherence was introduced (Schelter et al., 2009). Considering the two-dimensional vector

$$
\mathbf{X}_{i j}(\omega)=\left(\begin{array}{c}
\operatorname{Re}\left(\mathbf{A}_{i j}(\omega)\right) \\
\operatorname{Im}\left(\mathbf{A}_{i j}(\omega)\right)
\end{array}\right),
$$


with $\mathbf{X}_{i j}(\omega)^{\prime} \mathbf{X}_{i j}(\omega)=\left|\mathbf{A}_{i j}(\omega)\right|^{2}$ and

$$
\mathbf{A}(\omega)=I-\sum_{r=1}^{p} \mathbf{a}_{r} \mathrm{e}^{-\mathrm{i} \omega r}
$$

The corresponding estimator $\hat{\mathbf{X}}_{i j}(\omega)$ with $\hat{\mathbf{A}}_{i j}(\omega)$ substituted for $\mathbf{A}_{i j}(\omega)$ is asymptotically normally distributed with mean $\mathbf{X}_{i j}(\omega)$ and covariance matrix

$$
\mathbf{V}_{i j}(\omega)=\sum_{l, m=1}^{p} \operatorname{cov}\left(\left(\hat{\mathbf{a}}_{l}\right)_{i j},\left(\hat{\mathbf{a}}_{m}\right)_{i j}\right)\left(\begin{array}{ll}
\cos (l \omega) \cos (m \omega) & \cos (l \omega) \sin (m \omega) \\
\sin (l \omega) \cos (m \omega) & \sin (l \omega) \sin (m \omega)
\end{array}\right)
$$

Again, the covariance of the parameters $\operatorname{cov}\left(\left(\hat{\mathbf{a}}_{l}\right)_{i j},\left(\hat{\mathbf{a}}_{m}\right)_{i j}\right)$ can be substituted using the covariance matrix of the VAR process $\mathbf{R}$. Using $\mathbf{V}$, renormalised partial directed coherence

$$
\lambda_{i j}(\omega)=\mathbf{X}_{i j}(\omega)^{\prime} \mathbf{V}_{i j}^{-1}(\omega) \mathbf{X}_{i j}(\omega)
$$

is defined. A Granger-causal linear influence from $x_{j}$ to $x_{i}$ taking into account all other processes, can be rejected at frequency $\omega$, if $\lambda_{i j}(\omega)=0$. The critical value for an $\alpha$-significance level for $\lambda_{i j}(\omega)=0$ is given by $\chi_{2,1-\alpha}^{2}$ (Schelter et al., 2009). In this case, $\lambda_{i j}(\omega)$ does not depend on other outgoing links from $j$. Thus, a comparison of the strengths of influences at different frequencies is possible.

\section{Appendix B. Estimated parameters of AR[2]}

The auto-covariances of a one dimensional AR[2] process are related to its coefficients. For lag one

$$
\begin{aligned}
\langle x(t), x(t-1)\rangle & =\left\langle\left(\mathbf{a}_{1} x(t-1)+\mathbf{a}_{2} x(t-2)+\varepsilon(t)\right), x(t-1)\right\rangle \\
& =\mathbf{a}_{1}\langle x(t), x(t)\rangle+\mathbf{a}_{2}\langle x(t), x(t-1)\rangle \\
& =\frac{\mathbf{a}_{1}}{1-\mathbf{a}_{2}}\langle x(t), x(t)\rangle
\end{aligned}
$$


is obtained. Using Eqn. (B.1) the result for lag two

$$
\begin{aligned}
\langle x(t), x(t-2)\rangle & =\left\langle\left(\mathbf{a}_{1} x(t-1)+\mathbf{a}_{2} x(t-2)+\varepsilon(t)\right), x(t-2)\right\rangle \\
& =\mathbf{a}_{1}\langle x(t), x(t-1)\rangle+\mathbf{a}_{2}\langle x(t), x(t)\rangle \\
& =\left(\frac{\mathbf{a}_{1}^{2}}{1-\mathbf{a}_{2}}+\mathbf{a}_{2}\right)\langle x(t), x(t)\rangle \\
& =\frac{\mathbf{a}_{1}^{2}+\mathbf{a}_{2}-\mathbf{a}_{2}^{2}}{1-\mathbf{a}_{2}}\langle x(t), x(t)\rangle
\end{aligned}
$$

can be derived.

For the parameter estimates in the presence of observational noise this leads to

$$
\begin{aligned}
& \hat{\mathbf{a}}_{1}=\mathbf{a}_{1} \frac{(1+\mathrm{NSR})\left(1-\mathbf{a}_{2}\right)-\left(\mathbf{a}_{1}^{2}+\mathbf{a}_{2}-\mathbf{a}_{2}^{2}\right)}{(1+\mathrm{NSR})^{2}\left(1-\mathbf{a}_{2}\right)^{2}-\mathbf{a}_{1}^{2}} \\
& \hat{\mathbf{a}}_{2}=\frac{(1+\mathrm{NSR})\left(\mathbf{a}_{1}^{2}+\mathbf{a}_{2}-\mathbf{a}_{2}^{2}\right)\left(1-\mathbf{a}_{2}\right)-\mathbf{a}_{1}^{2}}{(1+\mathrm{NSR})^{2}\left(1-\mathbf{a}_{2}\right)^{2}-\mathbf{a}_{1}^{2}} .
\end{aligned}
$$

In the following we show that for NSR $>0$ the absolute values of the parameters are underestimated, i.e. $\left|\hat{\mathbf{a}}_{1}\right|<\left|\mathbf{a}_{1}\right|$ and $\left|\hat{\mathbf{a}}_{2}\right|<\left|\mathbf{a}_{2}\right|$. To obtain $\left|\hat{\mathbf{a}}_{1}\right|<\left|\mathbf{a}_{1}\right|$ it has to hold that

$$
\frac{(1+\mathrm{NSR})\left(1-\mathbf{a}_{2}\right)-\left(\mathbf{a}_{1}^{2}+\mathbf{a}_{2}-\mathbf{a}_{2}^{2}\right)}{(1+\mathrm{NSR})^{2}\left(1-\mathbf{a}_{2}\right)^{2}-\mathbf{a}_{1}^{2}}<1 .
$$

This is true since for a stationary $\operatorname{AR}[2] \mathbf{a}_{1}<\left(1-\mathbf{a}_{2}\right)$ and therefore

$$
\begin{aligned}
&(1+\mathrm{NSR})\left(1-\mathbf{a}_{2}\right)-\left(\mathbf{a}_{1}^{2}+\mathbf{a}_{2}-\mathbf{a}_{2}^{2}\right)<(1+\mathrm{NSR})^{2}\left(1-\mathbf{a}_{2}\right)^{2}-\mathbf{a}_{1}^{2} \\
& \Leftrightarrow 1+\mathrm{NSR}-\mathbf{a}_{2}-\mathbf{a}_{2} \mathrm{NSR}-\mathbf{a}_{1}^{2}-\mathbf{a}_{2}+\mathbf{a}_{2}^{2}<\left(1+2 \mathrm{NSR}+\mathrm{NSR}^{2}\right)\left(1-2 \mathbf{a}_{2}+\mathbf{a}_{2}^{2}\right)-\mathbf{a}_{1}^{2} \\
& \Leftrightarrow \operatorname{NSR}\left(1-\mathbf{a}_{2}\right)<\mathrm{NSR}(2+\mathrm{NSR})\left(1-2 \mathbf{a}_{2}+\mathbf{a}_{2}^{2}\right) \\
& \Leftrightarrow\left(1-\mathbf{a}_{2}\right)<(2+\mathrm{NSR})\left(1-\mathbf{a}_{2}\right)^{2} \\
& \Leftrightarrow 1<(2+\mathrm{NSR})\left(1-\mathbf{a}_{2}\right) .
\end{aligned}
$$

This holds since NSR $>0$ and $\mathbf{a}_{2}<0$ for an oscillatory AR[2]. 
Since $\mathbf{a}_{2}<0$ showing $\left|\hat{\mathbf{a}}_{2}\right|<\left|\mathbf{a}_{2}\right|$ can be achieved by demonstrating that

$$
\begin{aligned}
\frac{(1+\mathrm{NSR})\left(\mathbf{a}_{1}^{2}+\mathbf{a}_{2}-\mathbf{a}_{2}^{2}\right)\left(1-\mathbf{a}_{2}\right)-\mathbf{a}_{1}^{2}}{(1+\mathrm{NSR})^{2}\left(1-\mathbf{a}_{2}\right)^{2}-\mathbf{a}_{1}^{2}}>\mathbf{a}_{2} \\
\Leftrightarrow(1+\mathrm{NSR})\left(\mathbf{a}_{1}^{2}+\mathbf{a}_{2}-\mathbf{a}_{2}^{2}\right)\left(1-\mathbf{a}_{2}\right)-\mathbf{a}_{1}^{2}>\mathbf{a}_{2}\left((1+\mathrm{NSR})^{2}\left(1-\mathbf{a}_{2}\right)^{2}-\mathbf{a}_{1}^{2}\right) \\
\Leftrightarrow(1+\mathrm{NSR})\left(\mathbf{a}_{1}^{2}+\mathbf{a}_{2}-\mathbf{a}_{2}^{2}\right)\left(1-\mathbf{a}_{2}\right)>\mathbf{a}_{2}(1+\mathrm{NSR})^{2}\left(1-\mathbf{a}_{2}\right)^{2}+\mathbf{a}_{1}^{2}\left(1-\mathbf{a}_{2}\right) \\
\Leftrightarrow(1+\mathrm{NSR})\left(\mathbf{a}_{1}^{2}+\mathbf{a}_{2}-\mathbf{a}_{2}^{2}\right)>(1+\mathrm{NSR})^{2}\left(\mathbf{a}_{2}-\mathbf{a}_{2}^{2}\right)+\mathbf{a}_{1}^{2} \\
\Leftrightarrow \mathrm{NSR}\left(\mathbf{a}_{1}^{2}+\mathbf{a}_{2}-\mathbf{a}_{2}^{2}\right)>\mathrm{NSR}(2+\mathrm{NSR})\left(\mathbf{a}_{2}-\mathbf{a}_{2}^{2}\right) \\
\Leftrightarrow\left(\mathbf{a}_{1}^{2}+\mathbf{a}_{2}-\mathbf{a}_{2}^{2}\right)>(2+\mathrm{NSR})\left(\mathbf{a}_{2}-\mathbf{a}_{2}^{2}\right) \\
\Leftrightarrow 0>\mathbf{a}_{2}-\mathbf{a}_{2}^{2}-\mathbf{a}_{1}^{2}+\mathrm{NSR} \mathbf{a}_{2}-\mathrm{NSR} \mathbf{a}_{2}^{2} \\
\Leftrightarrow 0>(1+\mathrm{NSR}) \mathbf{a}_{2}-(1+\mathrm{NSR}) \mathbf{a}_{2}^{2}-\mathbf{a}_{1}^{2} \\
\Leftrightarrow 0>(1+\mathrm{NSR})\left(1-\mathbf{a}_{2}\right) \mathbf{a}_{2}-\mathbf{a}_{1}^{2} \quad(\mathrm{~B} .7)
\end{aligned}
$$

which holds as $\mathbf{a}_{2}<0$.

\section{Appendix C. Covariances of AR[2]}

In order to analyse the theoretical results when estimating an $\mathrm{AR}[2]$ process afflicted with observational noise, the covariances of the AR[2] process are needed. Here, these covariances are derived for two AR[2] processes with a unidirectional influence from $x_{2}$ onto $x_{1}$, i.e.

$$
\begin{aligned}
& x_{1}(t)=a_{1} x_{1}(t-1)+b_{1} x_{1}(t-2)+c x_{2}(t-1)+\varepsilon_{1}(t) \\
& x_{2}(t)=a_{2} x_{2}(t-1)+b_{2} x_{2}(t-2)+\varepsilon_{2}(t) .
\end{aligned}
$$

Since $x_{2}$ is independent of $x_{1}$, first, the auto-covariances of process $x_{2}$ are derived. For $\tau=0$

$$
\begin{aligned}
\left\langle x_{2}(t), x_{2}(t)\right\rangle & =\left\langle\left(a_{2} x_{2}(t-1)+b_{2} x_{2}(t-2)+\varepsilon_{2}(t)\right),\left(a_{2} x_{2}(t-1)+b_{2} x_{2}(t-2)+\varepsilon_{2}(t)\right)\right\rangle \\
& =a_{2}^{2}\left\langle x_{2}(t), x_{2}(t)\right\rangle+2 a_{2} b_{2}\left\langle x_{2}(t), x_{2}(t-1)\right\rangle+b_{2}^{2}\left\langle x_{2}(t), x_{2}(t)\right\rangle+\sigma_{2}^{2}
\end{aligned}
$$


is obtained. This can be solved if additionally the auto-covariance for $\tau=1$

$$
\begin{aligned}
\left\langle x_{2}(t), x_{2}(t-1)\right\rangle & =\left\langle x_{2}(t), x_{2}(t+1)\right\rangle \\
& =\left\langle x_{2}(t), a_{2} x_{2}(t)+b_{2} x_{2}(t-1)+\varepsilon_{2}(t+1)\right\rangle \\
& =a_{2}\left\langle x_{2}(t), x_{2}(t)\right\rangle+b_{2}\left\langle x_{2}(t), x_{2}(t-1)\right\rangle \\
\Leftrightarrow\left\langle x_{2}(t), x_{2}(t-1)\right\rangle & =\frac{a_{2}}{1-b_{2}}\left\langle x_{2}(t), x_{2}(t)\right\rangle
\end{aligned}
$$

is calculated. Inserting Eqn. (C.4) into Eqn. (C.3) leads to

$$
\begin{aligned}
& \left\langle x_{2}(t), x_{2}(t)\right\rangle=a_{2}^{2}\left\langle x_{2}(t), x_{2}(t)\right\rangle+\frac{2 a_{2}^{2} b_{2}}{1-b_{2}}\left\langle x_{2}(t), x_{2}(t)\right\rangle+b_{2}^{2}\left\langle x_{2}(t), x_{2}(t)\right\rangle+\sigma_{2}^{2} \\
& \Leftrightarrow\left\langle x_{2}(t), x_{2}(t)\right\rangle=\frac{\sigma_{2}^{2}}{1-\left(a_{2}^{2}+b_{2}^{2}+\frac{2 a_{2}^{2} b_{2}}{1-b_{2}}\right)} .
\end{aligned}
$$

The auto-covariances of $x_{1}$, for $\tau=0$ is

$$
\begin{aligned}
\left\langle x_{1}(t), x_{1}(t)\right\rangle= & \left\langle\left(a_{1} x_{1}(t-1)+b_{1} x_{1}(t-2)+c x_{2}(t-1)+\varepsilon_{1}(t)\right),\right. \\
& \left.\left(a_{1} x_{1}(t-1)+b_{1} x_{1}(t-2)+c x_{2}(t-1)+\varepsilon_{1}(t)\right)\right\rangle \\
= & a_{1}^{2}\left\langle x_{1}(t), x_{1}(t)\right\rangle+2 a_{1} b_{1}\left\langle x_{1}(t), x_{1}(t-1)\right\rangle \\
& +b_{1}^{2}\left\langle x_{1}(t), x_{1}(t)\right\rangle+\sigma_{1}^{2}+2 a_{1} c\left\langle x_{1}(t), x_{2}(t)\right\rangle \\
& +2 b_{1} c\left\langle x_{1}(t-1), x_{2}(t)\right\rangle+c^{2}\left\langle x_{2}(t), x_{2}(t)\right\rangle
\end{aligned}
$$

and for $\tau=1$

$$
\begin{aligned}
\left\langle x_{1}(t), x_{1}(t-1)\right\rangle & =\left\langle x_{1}(t), x_{1}(t+1)\right\rangle \\
& =\left\langle x_{1}(t),\left(a_{1} x_{1}(t)+b_{1} x_{1}(t-1)+c x_{2}(t)+\varepsilon_{1}(t+1)\right)\right\rangle \\
& =a_{1}\left\langle x_{1}(t), x_{1}(t)\right\rangle+b_{1}\left\langle x_{1}(t), x_{1}(t-1)\right\rangle+c\left\langle x_{1}(t), x_{2}(t)\right\rangle \\
& =\frac{a_{1}\left\langle x_{1}(t), x_{1}(t)\right\rangle+c\left\langle x_{1}(t), x_{2}(t)\right\rangle}{1-b_{1}}
\end{aligned}
$$


can be calculated. For the covariances, for $\tau=0$ we obtain

$$
\begin{aligned}
\left\langle x_{1}(t), x_{2}(t)\right\rangle= & \left\langle\left(a_{1} x_{1}(t-1)+b_{1} x_{1}(t-2)+c x_{2}(t-1)+\varepsilon_{1}(t)\right),\right. \\
& \left.\left(a_{2} x_{2}(t-1)+b_{2} x_{2}(t-2)+\varepsilon_{2}(t)\right)\right\rangle \\
= & a_{1} a_{2}\left\langle x_{1}(t), x_{2}(t)\right\rangle+a_{1} b_{2}\left\langle x_{1}(t), x_{2}(t-1)\right\rangle \\
& +b_{1} a_{2}\left\langle x_{1}(t-1), x_{2}(t)\right\rangle+b_{1} b_{2}\left\langle x_{1}(t), x_{2}(t)\right\rangle \\
& +c a_{2}\left\langle x_{2}(t), x_{2}(t)\right\rangle+c b_{2}\left\langle x_{2}(t), x_{2}(t-1)\right\rangle .
\end{aligned}
$$

For $\tau=1$

$$
\begin{aligned}
\left\langle x_{1}(t), x_{2}(t-1)\right\rangle & =\left\langle\left(a_{1} x_{1}(t-1)+b_{1} x_{1}(t-2)+c x_{2}(t-1)+\varepsilon_{1}(t)\right), x_{2}(t-1)\right\rangle \\
& =a_{1}\left\langle x_{1}(t), x_{2}(t)\right\rangle+b_{1}\left\langle x_{1}(t-1), x_{2}(t)\right\rangle+c\left\langle x_{2}(t), x_{2}(t)\right\rangle
\end{aligned}
$$

and

$$
\begin{aligned}
\left\langle x_{1}(t-1), x_{2}(t)\right\rangle & =\left\langle x_{1}(t-1),\left(a_{2} x_{2}(t-1)+b_{2} x_{2}(t-2)+\varepsilon_{2}(t)\right)\right\rangle \\
& =a_{2}\left\langle x_{1}(t), x_{2}(t)\right\rangle+b_{2}\left\langle x_{1}(t), x_{2}(t-1)\right\rangle
\end{aligned}
$$

are obtained. Inserting Eqn.(C.10) into Eqn. (C.9) leads to

$$
\begin{aligned}
\left\langle x_{1}(t), x_{2}(t-1)\right\rangle= & a_{1}\left\langle x_{1}(t), x_{2}(t)\right\rangle+b_{1} a_{2}\left\langle x_{1}(t), x_{2}(t)\right\rangle \\
& +b_{1} b_{2}\left\langle x_{1}(t), x_{2}(t-1)\right\rangle+c\left\langle x_{2}(t), x_{2}(t)\right\rangle \\
= & \frac{\left(a_{1}+b_{1} a_{2}\right)\left\langle x_{1}(t), x_{2}(t)\right\rangle+c\left\langle x_{2}(t), x_{2}(t)\right\rangle}{1-b_{1} b_{2}} .
\end{aligned}
$$


Inserting Eqn. (C.10) and then Eqn. (C.11) into Eqn. (C.8) yields

$$
\begin{aligned}
&\left\langle x_{1}(t), x_{2}(t)\right\rangle= a_{1} a_{2}\left\langle x_{1}(t), x_{2}(t)\right\rangle+b_{1} b_{2}\left\langle x_{1}(t), x_{2}(t)\right\rangle+c a_{2}\left\langle x_{2}(t), x_{2}(t)\right\rangle \\
&+c b_{2}\left\langle x_{2}(t), x_{2}(t-1)\right\rangle+a_{1} b_{2}\left\langle x_{1}(t), x_{2}(t-1)\right\rangle \\
&+b_{1} a_{2}^{2}\left\langle x_{1}(t), x_{2}(t)\right\rangle+b_{1} a_{2} b_{2}\left\langle x_{1}(t), x_{2}(t-1)\right\rangle \\
&= a_{1} a_{2}\left\langle x_{1}(t), x_{2}(t)\right\rangle+b_{1} b_{2}\left\langle x_{1}(t), x_{2}(t)\right\rangle+c a_{2}\left\langle x_{2}(t), x_{2}(t)\right\rangle \\
&+c b_{2}\left\langle x_{2}(t), x_{2}(t-1)\right\rangle+b_{1} a_{2} a_{2}\left\langle x_{1}(t), x_{2}(t)\right\rangle \\
&+\left(a_{1} b_{2}+b_{1} a_{2} b_{2}\right)\left\langle x_{1}(t), x_{2}(t-1)\right\rangle \\
&= a_{1} a_{2}\left\langle x_{1}(t), x_{2}(t)\right\rangle+b_{1} b_{2}\left\langle x_{1}(t), x_{2}(t)\right\rangle+c a_{2}\left\langle x_{2}(t), x_{2}(t)\right\rangle \\
&+c b_{2}\left\langle x_{2}(t), x_{2}(t-1)\right\rangle+b_{1} a_{2} a_{2}\left\langle x_{1}(t), x_{2}(t)\right\rangle \\
&+\left(a_{1} b_{2}+b_{1} a_{2} b_{2}\right) \frac{\left(a_{1}+b_{1} a_{2}\right)\left\langle x_{1}(t), x_{2}(t)\right\rangle+c\left\langle x_{2}(t), x_{2}(t)\right\rangle}{1-b_{1} b_{2}} \\
& c b_{2}\left\langle x_{2}(t), x_{2}(t-1)\right\rangle+\left(c a_{2}+\frac{\left(b_{1} a_{2} b_{2}+a_{1} b_{2}\right) c}{1-b_{1} b_{2}}\right)\left\langle x_{2}(t), x_{2}(t)\right\rangle \\
& 1-\left(a_{1} a_{2}+b_{1} b_{2}+b_{1} a_{2}^{2}+\frac{\left(b_{1} a_{2} b_{2}+a_{1} b_{2}\right)\left(a_{1}+b_{1} a_{2}\right)}{1-b_{1} b_{2}}\right)
\end{aligned}
$$

Finally, Eqs. (C.7) and (C.8) are inserted into Eqn. (C.6) leading to

$$
\begin{aligned}
\left\langle x_{1}(t), x_{1}(t)\right\rangle= & a_{1}^{2}\left\langle x_{1}(t), x_{1}(t)\right\rangle+2 a_{1} b_{1} \frac{a_{1}\left\langle x_{1}(t), x_{1}(t)\right\rangle+c\left\langle x_{1}(t), x_{2}(t)\right\rangle}{1-b_{1}} \\
& +b_{1}^{2}\left\langle x_{1}(t), x_{1}(t)\right\rangle+\sigma_{1}^{2}+2 a_{1} c\left\langle x_{1}(t), x_{2}(t)\right\rangle \\
& +2 b_{1} c\left\langle x_{1}(t-1), x_{2}(t)\right\rangle+c^{2}\left\langle x_{2}(t), x_{2}(t)\right\rangle \\
= & \left(a_{1}^{2} \frac{2 a_{1}^{2} b_{1}}{1-b_{1}}+b_{1}^{2}\right)\left\langle x_{1}(t), x_{1}(t)\right\rangle+\frac{2 a_{1} b_{1} c}{1-b_{1}}\left\langle x_{1}(t), x_{2}(t)\right\rangle \\
& +\sigma_{1}^{2}+\frac{2 a_{1} c-2 a_{1} b_{1} c}{1-b_{1}}\left\langle x_{1}(t), x_{2}(t)\right\rangle+2 b_{1} c\left\langle x_{1}(t-1), x_{2}(t)\right\rangle \\
& +c^{2}\left\langle x_{2}(t), x_{2}(t)\right\rangle \\
= & \frac{\sigma_{1}^{2}+\frac{2 a_{1} c}{1-b_{1}}\left\langle x_{1}(t), x_{2}(t)\right\rangle+2 b_{1} c\left\langle x_{1}(t-1), x_{2}(t)\right\rangle}{1-\left(a_{1}^{2}+b_{1}^{2}+\frac{2 a_{1}^{2} b_{1}}{1-b_{1}}\right)} \\
& +\frac{c^{2}\left\langle x_{2}(t), x_{2}(t)\right\rangle}{1-\left(a_{1}^{2}+b_{1}^{2}+\frac{2 a_{1}^{2} b_{1}}{1-b_{1}}\right)} .
\end{aligned}
$$

A recursive calculation of the covariances can thus be achieved. Starting with 
the auto-covariances for process $x_{2}$, equations have to be calculated in the following order

1. $\left\langle x_{2}(t), x_{2}(t)\right\rangle$ (Eqn. (C.5))

2. $\left\langle x_{2}(t), x_{2}(t-1)\right\rangle$ (Eqn. (C.4))

3. $\left\langle x_{1}(t), x_{2}(t)\right\rangle$ (Eqn. (C.12))

4. $\left\langle x_{1}(t), x_{2}(t-1)\right\rangle$ (Eqn. (C.11))

5. $\left\langle x_{1}(t-1), x_{2}(t)\right\rangle$ (Eqn. (C.10))

6. $\left\langle x_{1}(t), x_{1}(t)\right\rangle$ (Eqn. (C.13))

7. $\left\langle x_{1}(t), x_{1}(t-1)\right\rangle$ (Eqn. (C.7)).

\section{Appendix D. Incomplete Data Likelihood}

In order to assess the covariances of the estimated coefficients the second derivative of the incomplete data likelihood (Shumway and Stoffer, 2000)

$$
-\ln \mathrm{L}_{\vec{y}}(\boldsymbol{\Theta})=\frac{1}{2} \sum_{t=1}^{N} \log |\Sigma(t, \boldsymbol{\Theta})|+\frac{1}{2} \sum_{t=1}^{N} \epsilon(t, \boldsymbol{\Theta})^{T} \Sigma(t, \boldsymbol{\Theta})^{-1} \epsilon(t, \boldsymbol{\Theta})
$$

with innovations

$$
\begin{aligned}
\epsilon(t, \boldsymbol{\Theta}) & =\vec{y}(t)-\mathbf{C} \vec{u}(t \mid t-1) \\
\Sigma(t, \boldsymbol{\Theta}) & =\mathbf{C P}(t \mid t-1) \mathbf{C}^{T}+\mathbf{R}
\end{aligned}
$$

and

$$
\begin{aligned}
\mathbf{P}(t \mid t-1) & =\mathbf{A} \mathbf{P}(t-1 \mid t-1) \mathbf{A}^{T}+\mathbf{Q} \\
\mathbf{K}(t) & =\mathbf{P}(t \mid t-1) \mathbf{C}^{T}\left(\mathbf{C P}(t \mid t-1) \mathbf{C}^{T}+\mathbf{R}\right)^{-1} \\
\mathbf{P}(t \mid t) & =(1-\mathbf{K}(t) \mathbf{C}) \mathbf{P}(t \mid t-1) \\
\vec{u}(t \mid t-1) & =\mathbf{A} \vec{u}(t-1 \mid t-1) \\
\vec{u}(t \mid t) & =\vec{u}(t \mid t-1)+\mathbf{K}(t)(\vec{y}(t)-\mathbf{C} \vec{u}(t \mid t-1))
\end{aligned}
$$


has to be calculated. The first derivative is given by

$$
\begin{aligned}
-\frac{\partial \ln \mathrm{L}_{\vec{y}}(\boldsymbol{\Theta})}{\partial \Theta_{i}}= & \frac{1}{2} \sum_{t=1}^{N}\left(\operatorname{trace}\left(\Sigma(t, \boldsymbol{\Theta})^{-1} \frac{\partial \Sigma(t, \boldsymbol{\Theta})}{\partial \Theta_{i}}\right)\right. \\
& +\frac{1}{2} \sum_{t=1}^{N}\left(\frac{\partial \epsilon(t, \boldsymbol{\Theta})^{T}}{\partial \Theta_{i}} \Sigma(t, \boldsymbol{\Theta})^{-1} \epsilon(t, \boldsymbol{\Theta})+\epsilon(t, \boldsymbol{\Theta})^{T} \Sigma(t, \boldsymbol{\Theta})^{-1} \frac{\partial \epsilon(t, \boldsymbol{\Theta})}{\partial \Theta_{i}}\right. \\
& \left.-\epsilon(t, \boldsymbol{\Theta})^{T} \Sigma(t, \boldsymbol{\Theta})^{-1} \frac{\partial \Sigma(t, \boldsymbol{\Theta})}{\partial \Theta_{i}} \Sigma(t, \boldsymbol{\Theta})^{-1} \epsilon(t, \boldsymbol{\Theta})\right)
\end{aligned}
$$

with

$$
\begin{aligned}
\frac{\partial \epsilon(t, \boldsymbol{\Theta})}{\partial \Theta_{i}} & =-\mathbf{C} \frac{\partial \vec{u}(t \mid t-1)}{\partial \Theta_{i}} \\
\frac{\partial \Sigma(t, \boldsymbol{\Theta})}{\partial \Theta_{i}} & = \begin{cases}\mathbf{C} \frac{\partial \mathbf{P}(t \mid t-1)}{\partial \Theta_{i}} \mathbf{C}^{T}+e_{i} e_{j}^{T} & \text { for } \Theta_{i}=r_{i j} \\
\mathbf{C} \frac{\partial \mathbf{P}(t \mid t-1)}{\partial \Theta_{i}} \mathbf{C}^{T} & \text { else }\end{cases}
\end{aligned}
$$


and

$$
\begin{aligned}
\frac{\partial \mathbf{P}(t \mid t-1)}{\partial \Theta_{i}}= & e_{i} e_{j}^{T} \mathbf{P}(t-1 \mid t-1) \mathbf{A}^{T}+\mathbf{A} \frac{\partial \mathbf{P}(t-1 \mid t-1)}{\partial \Theta_{i}} \mathbf{A}^{T} \\
& +\mathbf{A} \mathbf{P}(t-1 \mid t-1) e_{j} e_{i}^{T} \text { for } \Theta_{i}=a_{i j} \\
\frac{\partial \mathbf{P}(t \mid t-1)}{\partial \Theta_{i}}= & \mathbf{A} \frac{\partial \mathbf{P}(t-1 \mid t-1)}{\partial \Theta_{i}} \mathbf{A}^{T}+e_{i} e_{j}^{T} \text { for } \Theta_{i}=q_{i j} \\
\frac{\partial \mathbf{P}(t \mid t-1)}{\partial \Theta_{i}}= & \mathbf{A} \frac{\partial \mathbf{P}(t-1 \mid t-1)}{\partial \Theta_{i}} \mathbf{A}^{T} \text { for } \Theta_{i}=r_{i j} \\
\frac{\partial \mathbf{K}(t)}{\partial \Theta_{i}}= & \left(1-\mathbf{P}(t \mid t-1) \mathbf{C}^{T}\left[\mathbf{C P}(t \mid t-1) \mathbf{C}^{T}+\mathbf{R}\right]^{-1} \mathbf{C}\right) \\
& \cdot \frac{\partial \mathbf{P}(t \mid t-1)}{\partial \Theta_{i}} \mathbf{C}^{T}\left[\mathbf{C P}(t \mid t-1) \mathbf{C}^{T}+\mathbf{R}\right]^{-1} \text { for } \Theta_{i}=a_{i j} \text { and } \Theta_{i}=q_{i j} \\
\frac{\partial \mathbf{K}(t)}{\partial \Theta_{i}}= & \frac{\partial \mathbf{P}(t \mid t-1)}{\partial \Theta_{i}} \mathbf{C}^{T}\left[\mathbf{C P}(t \mid t-1) \mathbf{C}^{T}+\mathbf{R}\right]^{-1} \\
& -\mathbf{P}(t \mid t-1) \mathbf{C}^{T}\left[\mathbf{C P}(t \mid t-1) \mathbf{C}^{T}+\mathbf{R}\right]^{-1} \\
& \cdot\left(\mathbf{C} \frac{\partial \mathbf{P}(t \mid t-1)}{\partial \Theta_{i}} \mathbf{C}^{T}+e_{i} e_{j}^{T}\right)\left[\mathbf{C P}(t \mid t-1) \mathbf{C}^{T}+\mathbf{R}\right]^{-1} \text { for } \Theta_{i}=r_{i j} \\
\frac{\partial \vec{u}(t \mid t-1)}{\partial \Theta_{i}}= & e_{i} e_{j}^{T} \vec{u}(t-1 \mid t-1)+\mathbf{A} \frac{\partial \vec{u}(t-1 \mid t-1)}{\partial \Theta_{i}} \text { for } \Theta_{i}=a_{i j} \\
\frac{\partial \Theta_{i}}{\partial(t \mid t-1)}= & \mathbf{A} \frac{\partial \vec{u}(t-1 \mid t-1)}{\partial \Theta_{i}} \text { for } \Theta_{i}=q_{i j} \text { and } \Theta_{i}=r_{i j} \\
\frac{\partial \vec{u}(t \mid t)}{\partial \Theta_{i}}= & (1-\mathbf{K}(t) \mathbf{C}) \frac{\partial \vec{u}(t \mid t-1)}{\partial \Theta_{i}}+\frac{\partial \mathbf{K}(t)}{\partial \Theta_{i}}(\vec{y}(t)-\mathbf{C} \vec{u}(t \mid t-1))
\end{aligned}
$$


Thus, the second derivative is

$$
\begin{aligned}
&-\frac{\partial^{2} \ln \mathrm{L}_{\vec{y}}(\boldsymbol{\Theta})}{\partial \Theta_{i} \partial \Theta_{k}}= \frac{1}{2} \sum_{t=1}^{N}\left(\operatorname { t r a c e } \left(\Sigma(t, \boldsymbol{\Theta})^{-1} \frac{\partial^{2} \Sigma(t, \boldsymbol{\Theta})}{\partial \Theta_{i} \partial \Theta_{k}}\right.\right. \\
&\left.\left.-\Sigma(t, \boldsymbol{\Theta})^{-1} \frac{\partial \Sigma(t, \boldsymbol{\Theta})}{\partial \Theta_{k}} \Sigma(t, \boldsymbol{\Theta})^{-1} \frac{\partial \Sigma(t, \boldsymbol{\Theta})}{\partial \Theta_{i}}\right)\right) \\
&+\frac{1}{2} \sum_{t=1}^{N}\left(\frac{\partial^{2} \epsilon(t, \boldsymbol{\Theta})^{T}}{\partial \Theta_{i} \partial \Theta_{k}} \Sigma(t, \boldsymbol{\Theta})^{-1} \epsilon(t, \boldsymbol{\Theta})\right. \\
&-\frac{\partial \epsilon(t, \boldsymbol{\Theta})^{T}}{\partial \Theta_{i}} \Sigma(t, \boldsymbol{\Theta})^{-1} \frac{\partial \Sigma(t, \boldsymbol{\Theta})}{\partial \Theta_{k}} \Sigma(t, \boldsymbol{\Theta})^{-1} \epsilon(t, \boldsymbol{\Theta}) \\
&+\frac{\partial \epsilon(t, \boldsymbol{\Theta})^{T}}{\partial \Theta_{i}} \Sigma(t, \boldsymbol{\Theta})^{-1} \frac{\partial \epsilon(t, \boldsymbol{\Theta})}{\partial \Theta_{k}} \\
&-\frac{\partial \epsilon(t, \boldsymbol{\Theta})^{T}}{\partial \Theta_{k}} \Sigma(t, \boldsymbol{\Theta})^{-1} \frac{\partial \Sigma(t, \boldsymbol{\Theta})}{\partial \Theta_{i}} \Sigma(t, \boldsymbol{\Theta})^{-1} \epsilon(t, \boldsymbol{\Theta}) \\
&+\epsilon(t, \boldsymbol{\Theta})^{T} \Sigma(t, \boldsymbol{\Theta})^{-1} \frac{\partial \Sigma(t, \boldsymbol{\Theta})}{\partial \Theta_{k}} \Sigma(t, \boldsymbol{\Theta})^{-1} \frac{\partial \Sigma(t, \boldsymbol{\Theta})}{\partial \Theta_{i}} \Sigma(t, \boldsymbol{\Theta})^{-1} \epsilon(t, \boldsymbol{\Theta}) \\
&-\epsilon(t, \boldsymbol{\Theta})^{T} \Sigma(t, \boldsymbol{\Theta})^{-1} \frac{\partial^{2} \Sigma(t, \boldsymbol{\Theta})}{\partial \Theta_{i} \partial \Theta_{k}} \Sigma(t, \boldsymbol{\Theta})^{-1} \epsilon(t, \boldsymbol{\Theta}) \\
&+\epsilon(t, \boldsymbol{\Theta})^{T} \Sigma(t, \boldsymbol{\Theta})^{-1} \frac{\partial \Sigma(t, \boldsymbol{\Theta})}{\partial \Theta_{i}} \Sigma(t, \boldsymbol{\Theta})^{-1} \frac{\partial \Sigma(t, \boldsymbol{\Theta})}{\partial \Theta_{k}} \Sigma(t, \boldsymbol{\Theta})^{-1} \epsilon(t, \boldsymbol{\Theta}) \\
&-\epsilon(t, \boldsymbol{\Theta})^{T} \Sigma(t, \boldsymbol{\Theta})^{-1} \frac{\partial \Sigma(t, \boldsymbol{\Theta})}{\partial \Theta_{i}} \Sigma(t, \boldsymbol{\Theta})^{-1} \frac{\partial \epsilon(t, \boldsymbol{\Theta})}{\partial \Theta_{k}} \\
&+\frac{\partial \epsilon(t, \boldsymbol{\Theta})^{T}}{\partial \Theta_{k}} \Sigma(t, \boldsymbol{\Theta})^{-1} \frac{\partial \epsilon(t, \boldsymbol{\Theta})}{\partial \Theta_{i}} \\
&-\epsilon(t, \boldsymbol{\Theta})^{T} \Sigma(t, \boldsymbol{\Theta})^{-1} \frac{\partial \Sigma(t, \boldsymbol{\Theta})}{\partial \Theta_{k}} \Sigma(t, \boldsymbol{\Theta})^{-1} \frac{\partial \epsilon(t, \boldsymbol{\Theta})}{\partial \Theta_{i}} \\
&\left.+\epsilon(t, \boldsymbol{\Theta})^{T} \Sigma(t, \boldsymbol{\Theta})^{-1} \frac{\partial^{2} \epsilon(t, \boldsymbol{\Theta})}{\partial \Theta_{i} \partial \Theta_{k}}\right) \\
&(D .22)
\end{aligned}
$$

with

$$
\begin{aligned}
\frac{\partial^{2} \epsilon(t, \boldsymbol{\Theta})}{\partial \Theta_{i} \partial \Theta_{k}} & =-\mathbf{C} \frac{\partial^{2} \vec{u}(t \mid t-1)}{\partial \Theta_{i} \partial \Theta_{k}} \\
\frac{\partial^{2} \Sigma(t, \boldsymbol{\Theta})}{\partial \Theta_{i} \partial \Theta_{k}} & =\mathbf{C} \frac{\partial^{2} \mathbf{P}(t \mid t-1)}{\partial \Theta_{i} \partial \Theta_{k}} \mathbf{C}^{T}
\end{aligned}
$$


and

$$
\begin{aligned}
\frac{\partial^{2} \mathbf{P}(t \mid t-1)}{\partial \Theta_{i} \partial \Theta_{k}}= & e_{i} e_{j}^{T} \frac{\partial \mathbf{P}(t-1 \mid t-1)}{\partial \Theta_{k}} \mathbf{A}^{T}+e_{i} e_{j}^{T} \mathbf{P}(t-1 \mid t-1) e_{l} e_{k}^{T} \\
& +e_{k} e_{l}^{T} \frac{\partial \mathbf{P}(t-1 \mid t-1)}{\partial \Theta_{i}} \mathbf{A}^{T}+\mathbf{A} \frac{\partial^{2} \mathbf{P}(t-1 \mid t-1)}{\partial \Theta_{i} \partial \Theta_{k}} \mathbf{A}^{T} \\
& +\mathbf{A} \frac{\partial \mathbf{P}(t-1 \mid t-1)}{\partial \Theta_{i}} e_{l} e_{k}^{T}+e_{k} e_{l}^{T} \mathbf{P}(t-1 \mid t-1) e_{j} e_{i}^{T} \\
& +\mathbf{A} \frac{\partial \mathbf{P}(t-1 \mid t-1)}{\partial \Theta_{k}} e_{j} e_{i}^{T} \text { for } \Theta_{i}=a_{i j} \text { and } \Theta_{k}=a_{k l} \\
\frac{\partial^{2} \mathbf{P}(t \mid t-1)}{\partial \Theta_{i} \partial \Theta_{k}}= & e_{i} e_{j}^{T} \frac{\partial \mathbf{P}(t-1 \mid t-1)}{\partial \Theta_{k}} \mathbf{A}^{T}+\mathbf{A} \frac{\partial^{2} \mathbf{P}(t-1 \mid t-1)}{\partial \Theta_{i} \partial \Theta_{k}} \mathbf{A}^{T} \\
& +\mathbf{A} \frac{\partial \mathbf{P}(t-1 \mid t-1)}{\partial \Theta_{k}} e_{j} e_{i}^{T} \text { for } \Theta_{i}=a_{i j} \text { and } \Theta_{k}=q_{k l} \text { or } r_{k l}
\end{aligned}
$$

$\frac{\partial^{2} \mathbf{P}(t \mid t-1)}{\partial \Theta_{i} \partial \Theta_{k}}=\mathbf{A} \frac{\partial^{2} \mathbf{P}(t-1 \mid t-1)}{\partial \Theta_{i} \partial \Theta_{k}} \mathbf{A}^{T}$ for $\Theta_{i}=q_{i j}$ or $r_{i j}$ and $\Theta_{k}=q_{k l}$ or $r_{k l}$

$$
\begin{aligned}
\frac{\partial^{2} \mathbf{K}(t)}{\partial \Theta_{i} \partial \Theta_{k}}= & {\left[\frac{\partial^{2} \mathbf{P}(t \mid t-1)}{\partial \Theta_{i} \partial \Theta_{k}}-\left(1-\mathbf{P}(t \mid t-1) \mathbf{C}^{T}\left[\mathbf{C P}(t \mid t-1) \mathbf{C}^{T}+\mathbf{R}\right]^{-1} \mathbf{C}\right)\right.} \\
& \cdot \frac{\partial \mathbf{P}(t \mid t-1)}{\partial \Theta_{i}} \mathbf{C}^{T}\left[\mathbf{C P}(t \mid t-1) \mathbf{C}^{T}+\mathbf{R}\right]^{-1} \mathbf{C} \frac{\partial \mathbf{P}(t \mid t-1)}{\partial \Theta_{k}} \\
& -\left(1-\mathbf{P}(t \mid t-1) \mathbf{C}^{T}\left[\mathbf{C P}(t \mid t-1) \mathbf{C}^{T}+\mathbf{R}\right]^{-1} \mathbf{C}\right) \\
& \cdot \frac{\partial \mathbf{P}(t \mid t-1)}{\partial \Theta_{k}} \mathbf{C}^{T}\left[\mathbf{C P}(t \mid t-1) \mathbf{C}^{T}+\mathbf{R}\right]^{-1} \mathbf{C} \frac{\partial \mathbf{P}(t \mid t-1)}{\partial \Theta_{i}} \\
& \left.-\mathbf{P}(t \mid t-1) \mathbf{C}^{T}\left[\mathbf{C P}(t \mid t-1) \mathbf{C}^{T}+\mathbf{R}\right]^{-1} \mathbf{C} \cdot \frac{\partial^{2} \mathbf{P}(t \mid t-1)}{\partial \Theta_{i} \partial \Theta_{k}}\right] \\
& \cdot \mathbf{C}^{T}\left[\mathbf{C P}(t \mid t-1) \mathbf{C}^{T}+\mathbf{R}\right]^{-1} \text { for } \Theta_{i}=a_{i j} \text { or } q_{i j} \text { and } \Theta_{k}=a_{k l} \text { or } q_{k l}
\end{aligned}
$$




$$
\begin{aligned}
\frac{\partial^{2} \mathbf{K}(t)}{\partial \Theta_{i} \partial \Theta_{k}}= & {\left[\frac{\partial^{2} \mathbf{P}(t \mid t-1)}{\partial \Theta_{i} \partial \Theta_{k}} \mathbf{C}^{T}-\frac{\partial \mathbf{P}(t \mid t-1)}{\partial \Theta_{i}} \mathbf{C}^{T}\left[\mathbf{C P}(t \mid t-1) \mathbf{C}^{T}+\mathbf{R}\right]^{-1} \mathbf{C} \frac{\partial \mathbf{P}(t \mid t-1)}{\partial \Theta_{k}} \mathbf{C}^{T}\right.} \\
& -\frac{\partial \mathbf{P}(t \mid t-1)}{\partial \Theta_{k}} \mathbf{C}^{T}\left[\mathbf{C P}(t \mid t-1) \mathbf{C}^{T}+\mathbf{R}\right]^{-1}\left(\mathbf{C} \frac{\partial \mathbf{P}(t \mid t-1)}{\partial \Theta_{i}} \mathbf{C}^{T}+e_{i} e_{j}^{T}\right) \\
& +\mathbf{P}(t \mid t-1) \mathbf{C}^{T}\left[\mathbf{C P}(t \mid t-1) \mathbf{C}^{T}+\mathbf{R}\right]^{-1} \mathbf{C} \frac{\partial \mathbf{P}(t \mid t-1)}{\partial \Theta_{k}} \mathbf{C}^{T} \\
& \cdot\left[\mathbf{C P}(t \mid t-1) \mathbf{C}^{T}+\mathbf{R}\right]^{-1}\left(\mathbf{C} \frac{\partial \mathbf{P}(t \mid t-1)}{\partial \Theta_{i}} \mathbf{C}^{T}+e_{i} e_{j}^{T}\right) \\
& -\mathbf{P}(t \mid t-1) \mathbf{C}^{T}\left[\mathbf{C P}(t \mid t-1) \mathbf{C}^{T}+\mathbf{R}\right]^{-1} \mathbf{C} \frac{\partial^{2} \mathbf{P}(t \mid t-1)}{\partial \Theta_{i} \partial \Theta_{k}} \mathbf{C}^{T} \\
& +\mathbf{P}(t \mid t-1) \mathbf{C}^{T}\left[\mathbf{C P}(t \mid t-1) \mathbf{C}^{T}+\mathbf{R}\right]^{-1}\left(\mathbf{C} \frac{\partial \mathbf{P}(t \mid t-1)}{\partial \Theta_{i}} \mathbf{C}^{T}+e_{i} e_{j}^{T}\right) \\
& \left.\cdot\left[\mathbf{C P}(t \mid t-1) \mathbf{C}^{T}+\mathbf{R}\right]^{-1} \mathbf{C} \frac{\partial \mathbf{P}(t \mid t-1)}{\partial \Theta_{k}} \mathbf{C}^{T}\right] \cdot\left[\mathbf{C P}(t \mid t-1) \mathbf{C}^{T}+\mathbf{R}\right]^{-1} \\
& \text { for } \Theta_{i}=r_{i j} \text { and } \Theta_{k}=a_{k l} \text { or } q_{k l}
\end{aligned}
$$

$$
\begin{aligned}
& \frac{\partial^{2} \mathbf{K}(t)}{\partial \Theta_{i} \partial \Theta_{k}}=\left[\frac{\partial^{2} \mathbf{P}(t \mid t-1)}{\partial \Theta_{i} \partial \Theta_{k}} \mathbf{C}^{T}\right. \\
& -\frac{\partial \mathbf{P}(t \mid t-1)}{\partial \Theta_{i}} \mathbf{C}^{T}\left[\mathbf{C P}(t \mid t-1) \mathbf{C}^{T}+\mathbf{R}\right]^{-1}\left(\mathbf{C} \frac{\partial \mathbf{P}(t \mid t-1)}{\partial \Theta_{k}} \mathbf{C}^{T}+e_{k} e_{l}^{T}\right) \\
& -\frac{\partial \mathbf{P}(t \mid t-1)}{\partial \Theta_{k}} \mathbf{C}^{T}\left[\mathbf{C P}(t \mid t-1) \mathbf{C}^{T}+\mathbf{R}\right]^{-1}\left(\mathbf{C} \frac{\partial \mathbf{P}(t \mid t-1)}{\partial \Theta_{i}} \mathbf{C}^{T}+e_{i} e_{j}^{T}\right) \\
& +\mathbf{P}(t \mid t-1) \mathbf{C}^{T}\left[\mathbf{C P}(t \mid t-1) \mathbf{C}^{T}+\mathbf{R}\right]^{-1}\left(\mathbf{C} \frac{\partial \mathbf{P}(t \mid t-1)}{\partial \Theta_{k}} \mathbf{C}^{T}+e_{k} e_{l}^{T}\right) \\
& \cdot\left[\mathbf{C P}(t \mid t-1) \mathbf{C}^{T}+\mathbf{R}\right]^{-1}\left(\mathbf{C} \frac{\partial \mathbf{P}(t \mid t-1)}{\partial \Theta_{i}} \mathbf{C}^{T}+e_{i} e_{j}^{T}\right) \\
& -\mathbf{P}(t \mid t-1) \mathbf{C}^{T}\left[\mathbf{C P}(t \mid t-1) \mathbf{C}^{T}+\mathbf{R}\right]^{-1} \mathbf{C} \frac{\partial^{2} \mathbf{P}(t \mid t-1)}{\partial \Theta_{i} \partial \Theta_{k}} \mathbf{C}^{T} \\
& +\mathbf{P}(t \mid t-1) \mathbf{C}^{T}\left[\mathbf{C P}(t \mid t-1) \mathbf{C}^{T}+\mathbf{R}\right]^{-1} \\
& \cdot\left(\mathbf{C} \frac{\partial \mathbf{P}(t \mid t-1)}{\partial \Theta_{i}} \mathbf{C}^{T}+e_{i} e_{j}^{T}\right)\left[\mathbf{C P}(t \mid t-1) \mathbf{C}^{T}+\mathbf{R}\right]^{-1} \\
& \left.\cdot\left(\mathbf{C} \frac{\partial \mathbf{P}(t \mid t-1)}{\partial \Theta_{k}} \mathbf{C}^{T}+e_{k} e_{l}^{T}\right)\right] \cdot\left[\mathbf{C P}(t \mid t-1) \mathbf{C}^{T}+\mathbf{R}\right]^{-1}
\end{aligned}
$$

for $\Theta_{i}=r_{i j}$ and $\Theta_{k}=r_{k l}$ 


$$
\begin{aligned}
\frac{\partial^{2} \mathbf{P}(t \mid t)}{\partial \Theta_{i} \partial \Theta_{k}}= & (1-\mathbf{K}(t) \mathbf{C}) \frac{\partial^{2} \mathbf{P}(t \mid t-1)}{\partial \Theta_{i} \partial \Theta_{k}}-\frac{\partial \mathbf{K}(t)}{\partial \Theta_{k}} \mathbf{C} \frac{\partial \mathbf{P}(t \mid t-1)}{\partial \Theta_{i}} \\
& -\frac{\partial^{2} \mathbf{K}(t)}{\partial \Theta_{i} \partial \Theta_{k}} \mathbf{C P}(t \mid t-1)-\frac{\partial \mathbf{K}(t)}{\partial \Theta_{i}} \mathbf{C} \frac{\partial \mathbf{P}(t \mid t-1)}{\partial \Theta_{k}} \\
\frac{\partial^{2} \vec{u}(t \mid t-1)}{\partial \Theta_{i} \partial \Theta_{k}}= & e_{k} e_{l}^{T} \frac{\partial \vec{u}(t-1 \mid t-1)}{\partial \Theta_{i}}+\mathbf{A} \frac{\partial^{2} \vec{u}(t-1 \mid t-1)}{\partial \Theta_{i} \partial \Theta_{k}} \\
& +e_{i} e_{j}^{T} \frac{\partial \vec{u}(t-1 \mid t-1)}{\partial \Theta_{k}} \text { for } \Theta_{i}=a_{i j} \text { and } \Theta_{k}=a_{k l} \\
\frac{\partial^{2} \vec{u}(t \mid t-1)}{\partial \Theta_{i} \partial \Theta_{k}}= & \mathbf{A} \frac{\partial^{2} \vec{u}(t-1 \mid t-1)}{\partial \Theta_{i} \partial \Theta_{k}}+e_{i} e_{j}^{T} \frac{\partial \vec{u}(t-1 \mid t-1)}{\partial \Theta_{k}} \text { for } \Theta_{i}=a_{i j} \text { and } \Theta_{k}=q_{k l} \text { or } r_{k l}
\end{aligned}
$$

$\frac{\partial^{2} \vec{u}(t \mid t-1)}{\partial \Theta_{i} \partial \Theta_{k}}=\mathbf{A} \frac{\partial^{2} \vec{u}(t-1 \mid t-1)}{\partial \Theta_{i} \partial \Theta_{k}}$ for $\Theta_{i}=q_{i j}$ or $r_{i j}$ and $\Theta_{k}=q_{k l}$ or $r_{k l}$

$$
\begin{aligned}
\frac{\partial^{2} \vec{u}(t \mid t)}{\partial \Theta_{i} \partial \Theta_{k}}= & (1-\mathbf{K}(t) \mathbf{C}) \frac{\partial^{2} \vec{u}(t \mid t-1)}{\partial \Theta_{i} \partial \Theta_{k}}-\frac{\partial \mathbf{K}(t)}{\partial \Theta_{k}} \mathbf{C} \frac{\partial \vec{u}(t \mid t-1)}{\partial \Theta_{i}} \\
& +\frac{\partial^{2} \mathbf{K}(t)}{\partial \Theta_{i} \partial \Theta_{k}}(\vec{y}(t)-\mathbf{C} \vec{u}(t \mid t-1))-\frac{\partial \mathbf{K}(t)}{\partial \Theta_{i}} \mathbf{C} \frac{\partial \vec{u}(t \mid t-1)}{\partial \Theta_{k}}
\end{aligned}
$$

